\title{
GRB 161219B/SN 2016jca: A low-redshift gamma-ray burst supernova powered by radioactive heating
}

\author{
Z. Cano ${ }^{1}$, L. Izzo ${ }^{1}$, A. de Ugarte Postigo ${ }^{1,2}$, C. C. Thöne ${ }^{1}$, T. Krühler ${ }^{3}$, K. E. Heintz ${ }^{4,2}$, D. Malesani ${ }^{2,5}$, S. Geier ${ }^{6,7}$,
} C. Fuentes ${ }^{8,9}$, T.-W. Chen ${ }^{10}$, S. Covino ${ }^{11}$, V. D'Elia ${ }^{12,13}$, J. P. U. Fynbo ${ }^{2}$, P. Goldoni ${ }^{14}$, A. Gomboc ${ }^{15}$, J. Hjorth ${ }^{2}$, P. Jakobsson ${ }^{4}$, D. A. Kann ${ }^{1}$, B. Milvang-Jensen ${ }^{2}$, G. Pugliese ${ }^{16}$, R. Sánchez-Ramírez ${ }^{1}$, S. Schulze ${ }^{17}$, J. Sollerman ${ }^{18}$, N. R. Tanvir ${ }^{19}$, and K. Wiersema ${ }^{19}$

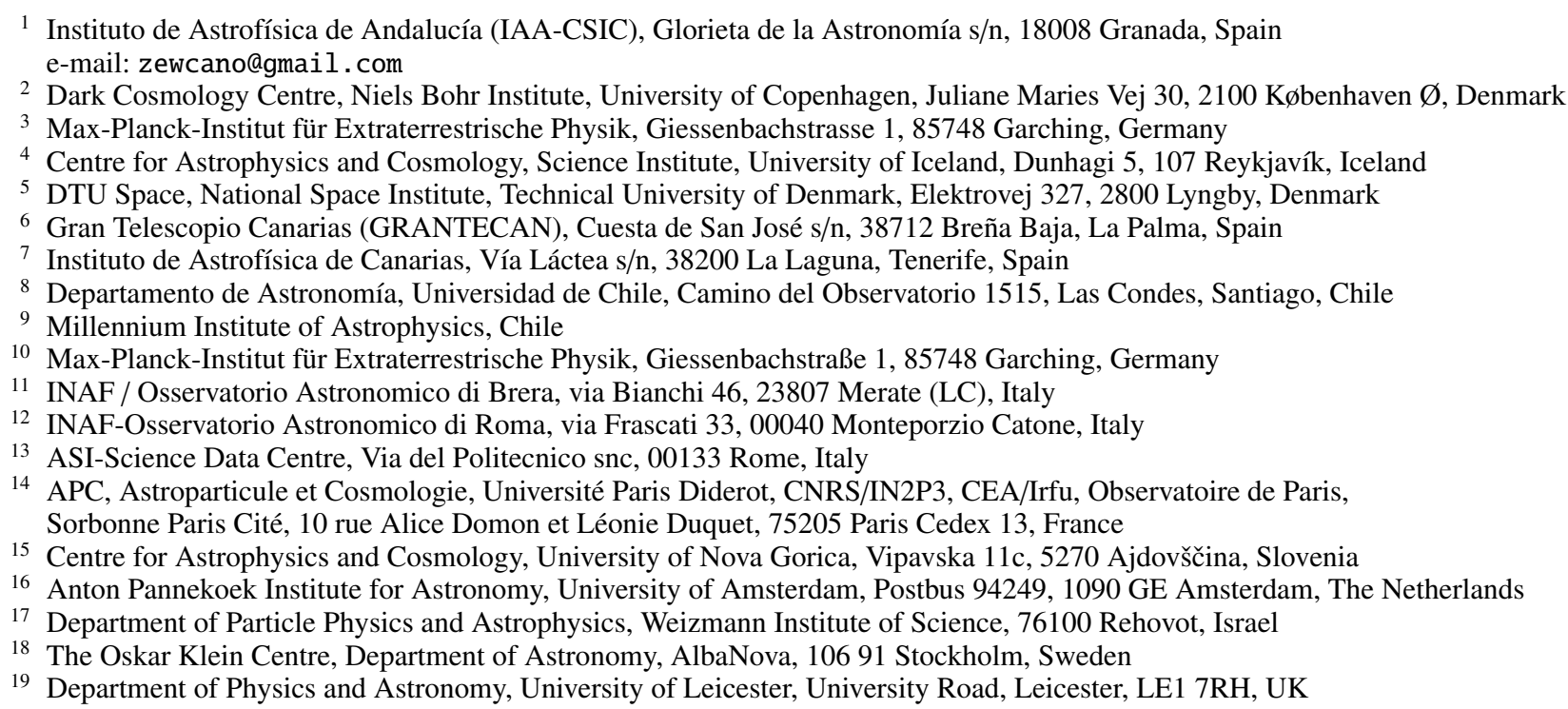

Received 18 April 2017 / Accepted 5 July 2017

\begin{abstract}
Since the first discovery of a broad-lined type Ic supernova (SN) with a long-duration gamma-ray burst (GRB) in 1998, fewer than fifty GRB-supernovae (SNe) have been discovered. The intermediate-luminosity Swift GRB 161219B and its associated supernova SN 2016jca, which occurred at a redshift of $z=0.1475$, represents only the seventh GRB-SN to have been discovered within $1 \mathrm{Gpc}$, and hence provides an excellent opportunity to investigate the observational and physical properties of these very elusive and rare type of SN. As such, we present optical to near-infrared photometry and optical spectroscopy of GRB 161219B and SN 2016jca, spanning the first three months since its discovery. GRB 161219B exploded in the disk of an edge-on spiral galaxy at a projected distance of $3.4 \mathrm{kpc}$ from the galactic centre. GRB 161219B itself is an outlier in the $E_{\mathrm{p}, \mathrm{i}}-E_{\gamma, \text { iso }}$ plane, while SN 2016jca had a rest-frame, peak absolute $V$-band magnitude of $M_{V}=-19.0 \pm 0.1$, which it reached after $12.3 \pm 0.7$ rest-frame days. We find that the bolometric properties of SN 2016jca are inconsistent with being powered solely by a magnetar central engine, and demonstrate that it was likely powered exclusively by energy deposited by the radioactive decay of nickel and cobalt into their daughter products, which were nucleosynthesised when its progenitor underwent core collapse. We find that $0.22 \pm 0.08 M_{\odot}$ of nickel is required to reproduce the peak luminosity of SN 2016jca, and we constrain an ejecta mass of $5.8 \pm 0.3 M_{\odot}$ and a kinetic energy of $5.1 \pm 0.8 \times 10^{52} \mathrm{erg}$. Finally, we report on a chromatic, pre-maximum bump in the $g$-band light curve, and discuss its possible origin.
\end{abstract}

Key words. gamma-ray burst: individual: GRB 161219B - supernovae: individual: SN 2016jca - gamma-ray burst: general supernovae: general

\section{Introduction}

The connection between long-duration gamma-ray bursts (GRBs) and broad-lined type Ic supernovae (SNe IcBL) is now firmly established: i.e. the "GRB-SN connection" (e.g. Woosley \& Bloom 2006; Cano et al. 2017). GRB-SNe are intrinsically rare; in the two decades since the association between
GRB 980425 and SN 1998bw (Galama et al. 1998; Patat et al. 2001), fewer than 50 events have been detected (Cano et al. 2017) to varying degrees of confidence (Hjorth \& Bloom 2012), over a wide range of redshifts $(z=0.0087$ for GRB 980425, Li et al. 2014; to $z=1.0585$ for GRB 000911, Price et al. 2002). Indeed only six confirmed GRB-SNe have been detected within $1 \mathrm{Gpc}(z \leq 0.2)$, and only one of 
these was a high-luminosity $\mathrm{GRB}^{1}$ : GRB $030329 / \mathrm{SN} 2003 \mathrm{dh}$ (Hjorth et al. 2003; Stanek et al. 2003; Matheson et al. 2003); while the remainder were an intermediate-luminosity GRB: GRB 130702A/SN 2013dx (D'Elia et al. 2015; Toy et al. 2016; Volnova et al. 2017); and low-luminosity GRBs: GRB 980425/SN 1998bw (Galama et al. 1998; Patat et al. 2001); GRB 031203/SN 20031w (Malesani et al. 2004); GRB 060218/SN 2006aj, (Pian et al. 2006; Mazzali et al. 2006); and GRB 100316D/SN 2010bh (Cano et al. 2011b; Bufano et al. 2012; Olivares et al. 2012).

Despite their rarity, many aspects regarding their physical properties have been established. Their explosion mechanism is thought to be driven by the compact object that forms at the time of core-collapse: either a stellar-mass black hole (BH; Woosley 1993; MacFadyen \& Woosley 1999) or a rapidly rotating neutron-star with an exceptionally large magnetic field (a magnetar; Usov 1992; Thompson \& Duncan 1993; Bucciantini et al. 2007). It is thought that these "central engines" lead to the explosion of the star, rather than the conventional neutrino-driven explosion mechanism (e.g. Sukhbold et al. 2016). From modelling observations of GRB$\mathrm{SNe}$, it has been demonstrated that their kinetic energies cluster around a value of $2-2.5 \times 10^{52}$ erg (Mazzali et al. 2014), which may indicate the formation of a magnetar central engine. However, if magnetars are formed in the majority of GRB-SNe, they are unlikely to be the dominant source of energy that powers their luminosity; instead they are very likely powered by radioactive heating (Cano et al. 2016). Next, GRBSNe have a luminosity-stretch/decline relationship (Cano 2014; Li \& Hjorth 2014; Cano et al. 2014c) analogous to that of type Ia SNe (Phillips 1993), which implies their use as cosmological probes (Li et al. 2014; Cano et al. 2014c).

While many aspects of the GRB-SN connection have been determined, over the years even more complex GRB-SN phenomenology has been observed. The connection between ultralong duration (Levan et al. 2014) GRB 111209A and its associated SN 2011kl (Greiner et al. 2015; Kann et al. 2016) is one such example. SN $2011 \mathrm{kl}$ is peculiar in many ways, including being exceptionally luminous (the most luminous GRB$\mathrm{SN}$ to date), and whose peak optical spectrum was quite flat and featureless, where the undulations typical of GRB-SNe were conspicuously absent. Instead, the shape of the spectrum more resembled that of superluminous supernovae (SLSNe; Quimby et al. 2011; Gal-Yam 2012), thus suggesting a link between these two types of luminous $\mathrm{SNe}$, perhaps in terms of their explosion mechanism and/or processes powering the $\mathrm{SNe}$ themselves. An interpretation of both the spectrum and the bolometric light curve (LC) of SN 2011kl led to the conclusion that the GRB and SN were both driven by a magnetar central engine, and radioactive heating played a minor, perhaps even negligible role in powering its luminosity (Greiner et al. 2015; Metzger et al. 2015; Bersten et al. 2016; Cano et al. 2016; Gompertz \& Fruchter 2017). A magnetar central engine has been inferred for several type Ic SLSNe (Chatzopoulos et al. 2011; Inserra et al. 2013; Nicholl et al. 2013; Chen et al. 2015), suggesting at least one common theme between these extreme stellar explosions. As the luminosity of a magnetar-powered SN is directly related to how long the central engine is active, where

\footnotetext{
1 Definitions for the isotropic-equivalent luminosity in $\gamma$-rays, $L_{\gamma, \text { iso }}$, e.g. Bromberg et al. (2011), Hjorth (2013), Cano et al. (2017), are: lowluminosity GRB: $L_{\gamma, \text { iso }}<10^{48.5} \mathrm{erg} \mathrm{s}^{-1}$; intermediate-luminosity GRB: $10^{48.5} \leq L_{\gamma \text {,iso }} \leq 10^{49.5} \mathrm{erg} \mathrm{s}^{-1}$; and high-luminosity GRB: $L_{\gamma, \text { iso }}>$
$10^{49.5} \mathrm{erg} \mathrm{s}^{-1}$.
}

central engines with longer durations give rise to brighter $\mathrm{SNe}$, the key difference between the magnetar's properties in a SLSNe relative to $\mathrm{SN} 2011 \mathrm{kl}$ is the spin-down timescale, which is of order several weeks to months for SLSNe, but was less than a week for SN 2011kl (Cano et al. 2016). This naturally explains why SN 2011kl, though more luminous than all other GRB-SNe, was not as luminous as SLSNe-I.

In this paper we take a close look at GRB 161219B and its associated SN 2016jca. GRB 161219B is only the seventh GRB to be detected at $z<0.2$, and the closest longduration GRB to be detected by Swift since GRB 100316D (Starling et al. 2011). As such, this event represents a rare opportunity to determine the detailed properties of a nearby GRBSN. GRB 161219B was detected by the Burst Alert Telescope (BAT) aboard Swift (Gehrels et al. 2004) at 18:48:39 UT on the 19th of December, 2016 (D'Ai et al. 2016). The BAT light curve showed a single-peaked structure with a duration of $\sim 10 \mathrm{~s}$. The enhanced X-Ray Telescope (XRT) location was found to be RA, Dec (J2000) $06^{\mathrm{h}} 06^{\mathrm{m}} 51.36^{\mathrm{s}}-26^{\mathrm{d}} 47^{\prime} 29.9^{\prime \prime}$, with an uncertainty of $1.7^{\prime \prime}$ (Beardmore et al. 2016). The GRB was also detected by Konus-Wind (Frederiks et al. 2016), who measured a duration of roughly 10 seconds, and by POLAR (Xiao et al. 2016), who measured a duration of $T_{90}=4.0 \pm 0.5 \mathrm{~s}$. The optical and near-infrared (NIR) afterglow (AG) was detected by many ground-based facilities (Buckley et al. 2016; Fong \& Milne 2016; Fujiwara et al. 2016; Guidorzi et al. 2016; Krühler et al. 2016; Marshall \& D'Ai 2016; Martin-Carrillo et al. 2016; Mazaeva et al. 2016). The AG was also detected at sub-mm (Laskar et al. 2016) and radio (Alexander et al. 2016; Nayana \& Chandra 2016) frequencies. The redshift was determined from an optical spectrum of the AG to be $z=0.1475$ (Tanvir et al. 2016; de Ugarte Postigo et al. 2016). Spectroscopic identification of its associated SN 2016jca was first given by de Ugarte Postigo et al. (2016), which was later verified by a photometric SN-"bump" by Volnova et al. (2017) and additional spectroscopy by (Chen et al. 2017). Finally, an in-depth investigation of the $\mathrm{SN}$ is presented in Ashall et al. (2017), who concluded that a magnetar central engine likely powered SN 2016jca, which is at odds to our results here, where we demonstrate that the $\mathrm{SN}$ was likely powered in part, or perhaps exclusively, by energy deposited by the radioactive decay of nickel and cobalt into their daughter products.

All data presented here have been corrected for foreground extinction using the dust maps of Schlegel et al. (1998) as revised by Schlafly \& Finkbeiner (2011). We have assumed a generic $\Lambda$-CDM cosmological model with $H_{0}=$ $70 \mathrm{~km} \mathrm{~s}^{-1} \mathrm{Mpc}^{-1}, \Omega_{\mathrm{M}}=0.27$, and $\Omega_{\Lambda}=0.73$. The respective forward-shock afterglow (AG) decay and energy spectral indices $\alpha$ and $\beta$ are defined by $f_{v} \propto\left(t-t_{0}\right)^{-\alpha} v^{-\beta}$, where $t_{0}$ is the time at which the GRB triggered Swift-BAT, and $v$ is the observed frequency.

The paper is organised as: in Sect. 2 we present our optical and NIR photometry, including our data-reduction \& calibration procedures. In Sect. 3 we discuss the high-energy ( $\gamma$ - and X-ray) properties of GRB 161219B. We present our investigation of the optical/NIR properties of the AG (Sect. 6) and accompanying SN (Sect. 7), which follows our analysis of the time-resolved X-ray to NIR spectral energy distribution (SED) of GRB 161219B (Sect. 4). In Sect. 8 we model a quasi-bolometric LC constructed from our de-reddened (foreground and host), host- and AG-subtracted observations to determine whether radioactiveheating or a magnetar is responsible for powering its luminosity. In Sect. 9 we discuss the intriguing presence of a chromatic premaximum bump present only in the $g$-band LC of SN 2016jca. 

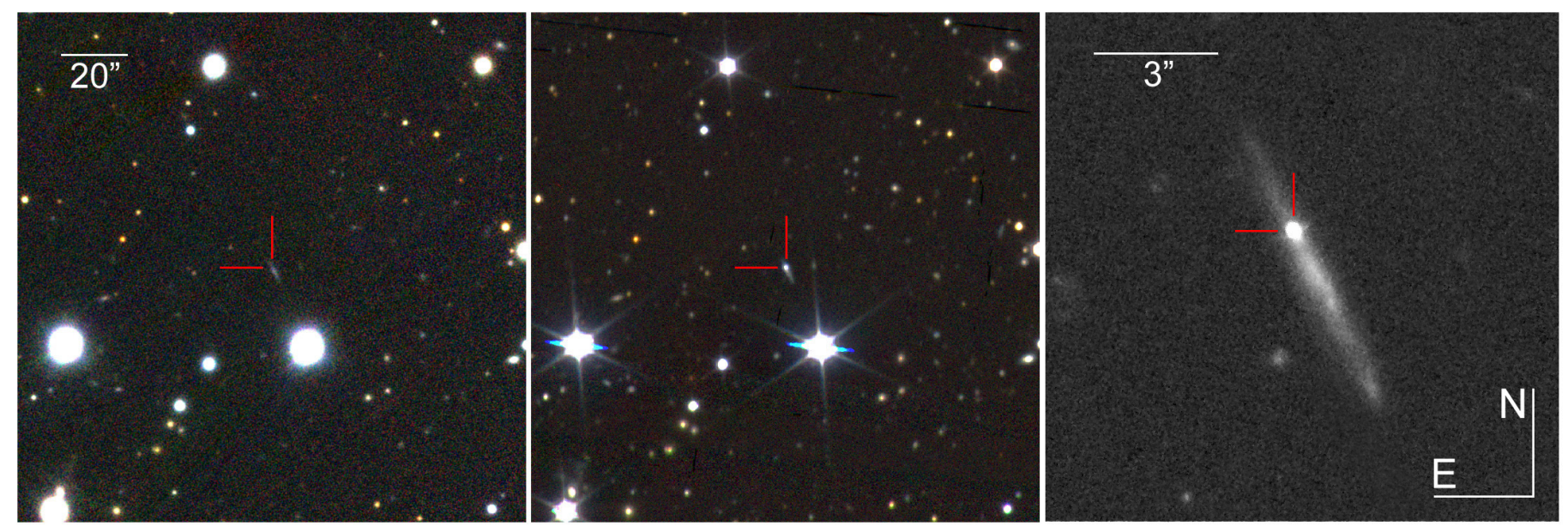

Fig. 1. Left: RGB composite Pan-STARRS1 pre-imaging of the host galaxy. Centre: a GTC image (RGB) showing the supernova starting to emerge on the 27th of December, 2016. Right: HST WFC/UVIS F200LP image of the host galaxy and optical transient from the January 16 th, 2017.

The observational and physical properties of its host galaxy are presented in Sect. 10, and finally a discussion and our conclusions are given in Sect. 11.

\section{Data reduction, photometry, and spectroscopy}

\subsection{Photometry}

We obtained optical and NIR observations with three groundbased telescopes: $g^{\prime} r^{\prime} i^{\prime} z^{\prime} J H K_{\mathrm{S}}$ imaging with the Gamma-Ray burst Optical/Near-infrared Detector (GROND; Greiner et al. 2008) mounted on the MPG 2.2 m telescope on La Silla, Chile; grizJH imaging with the $2.5 \mathrm{~m}$ Nordic Optical Telescope (NOT), and grizJHK imaging with the $10.4 \mathrm{~m}$ Gran Telescopio Canarias (GTC) telescope (OSIRIS and CIRCE), both located on La Palma, Spain. Image reduction of the GTC, NOT and GROND (Krühler et al. 2008) data was performed using standard techniques in IRAF $^{2}$, while those obtained with CIRCE (Garner et al. 2014) were reduced using custom codes written in IDL.

The optical griz images were calibrated to the Panoramic Survey Telescope and Rapid Response System (Pan-STARRS1; Chambers et al. 2016; Flewelling et al. 2016). Twenty-three Pan-STARRS1 reference stars in the GRB field-of-view were chosen, and zeropoints were calculated between the instrumental magnitudes and catalog values. The NIR images $(J H K)$ were calibrated to the 2MASS catalog (Kleinmann et al. 1994) also using a zeropoint calculation. Due to differences between the GROND and Pan-STARRS1 filters, an additional zeropoint correction was applied to the $g$ - and $z$-band GROND images following the prescription in Finkbeiner et al. (2016; see their Table 2 and Eq. (1)).

Inspection of the images reveals that GRB 161219B occurred in an apparent edge-on spiral galaxy (Sect. 10; see Fig. 1), meaning that the photometry of the optical transient (OT) is polluted by flux from the underlying host. In order to provide a consistent analysis of the photometric evolution of the OT, all aperture photometry was performed using an aperture with a 2 "'2 radius centred on the position of the OT.

2 IRAF is distributed by the National Optical Astronomy Observatory, which is operated by the Association of Universities for Research in Astronomy, Inc., under cooperative agreement with the National Science Foundation.
The position of the OT was determined via two methods: (1) for the first night of imaging obtained on each telescope (i.e. when the OT was brightest), we used the IRAF routine centroid within the DIGIPHOT/DAOPHOT package and compared the determined centroid position in the wcs-corrected images among the different telescopes; next (2) we used the Pan-STARRS1 images in griz as templates for the image-subtraction technique, which was performed using an adaptation of the original ISIS program (Alard \& Lupton 1998; Alard 2000) that was developed for Hubble Space Telescope (HST) SN surveys by Strolger et al. (2004), and employed in other GRB-SN studies by our group (Cano et al. 2011a, 2014b, 2015). We performed image subtraction on the same epoch as method (1), and used centroid to find the position of the OT in the subtracted image, and related the $x, y$ position of the OT in the subtracted image to the RA, Dec positions in the original, wcs-corrected image. We then took the average of all values, finding a position of GRB 161219B of RA, Dec (J2000) $06^{\mathrm{h}} 06^{\mathrm{m}} 51.412^{\mathrm{s}}(29)-26^{\mathrm{d}} 47^{\prime} 29.49^{\prime \prime}(15)$. The quoted errors are statistical only.

Our monitoring campaign of GRB 161219B/SN 2016jca finished at the end of February 2017 when it was no longer visible from La Palma. As such, we were not able to obtain late-time images of the host galaxy in each filter obtained by each telescope/detector to use as templates for image subtraction. Initially we intended to use the Pan-STARRS1 images in griz as templates for the image subtraction technique, and subtract them from the griz data obtained with the GTC, NOT and GROND. However, initial tests reveals that the subtraction produced magnitudes in a given filter for data obtained on the date with the GTC/NOT/GROND that varied by several tenths of a magnitude. This unsatisfactory result led us to abandon this method. Instead we quantified the amount of host flux present in the images by performing aperture photometry on the Pan-STARRS1 images using an aperture of $2^{\prime \prime} .2$ centred on the position quoted above, and then subtracted this flux away from the GTC/NOT/GROND fluxes. In this sense the data presented in Fig. 5 (right panel) are host subtracted via the flux-subtraction technique (Cano 2014; Cano et al. 2017). We note that we will obtain template images in all filters with each telescope once the GRB field becomes visible again, which will be used in the image-subtraction technique in a future study. 
Table 1. GRB 161219B/SN 2016jca: vital statistics.

\begin{tabular}{ll}
\hline \hline GRB $161219 \mathrm{~B} / \mathrm{SN} 2016 \mathrm{jca}$ & Ref. \\
\hline $\mathrm{RA}(\mathrm{J} 2000)=06^{\mathrm{h}} 06^{\mathrm{m}} 51.412^{\mathrm{s}}$ & this work \\
$\operatorname{Dec}(\mathrm{J} 2000)=-26^{\mathrm{d}} 47^{\prime} 29.49^{\prime \prime}$ & this work \\
$z=0.1475$ & Tanvir et al. (2016) \\
$d_{\mathrm{L}}^{*}=700 \mathrm{Mpc}$ & this work \\
$\mu^{*}=39.22 \mathrm{mag}$ & this work \\
$E(B-V)_{\text {fore }}=0.0281 \pm 0.0002 \mathrm{mag}$ & Schlafly \& Finkbeiner (2011) \\
$E(B-V)_{\text {host }}=0.017 \pm 0.012 \mathrm{mag}$ & this work \\
$t_{90}=6.9 \mathrm{~s}$ & this work \\
$E_{\gamma, \text { iso,rest }}=\left(8.50_{-3.75}^{+8.46}\right) \times 10^{49} \mathrm{erg}$ & this work \\
$E_{\gamma, \mathrm{p}, \text { rest }}=62.9_{-19.9}^{+47.0} \mathrm{keV}$ & this work \\
$L_{\gamma}=\left(1.41_{-0.62}^{+1.41}\right) \times 10^{49} \mathrm{erg} \mathrm{s}{ }^{-1}$ & this work \\
$v_{\mathrm{ph}, \text { peak }}=29700 \pm 1500 \mathrm{~km} \mathrm{~s}{ }^{-1}$ & this work, based on Fe II $\lambda 5169$ \\
$M_{\mathrm{Ni}}=0.22 \pm 0.08 M_{\odot}$ & this work \\
$M_{\mathrm{ej}}=5.8 \pm 0.3 M_{\odot}$ & this work \\
$E_{\mathrm{K}}=(5.1 \pm 0.8) \times 10^{52} \mathrm{erg}$ & this work \\
\hline
\end{tabular}

Notes. ${ }^{*}$ Calculated using $H_{0}=70 \mathrm{~km} \mathrm{~s}^{-1} \mathrm{Mpc}^{-1}, \Omega_{\mathrm{M}}=0.3, \Omega_{\Lambda}=0.7$.

\subsection{Spectroscopy}

We obtained eight epochs of spectroscopy ${ }^{3}$ of GRB 161219B and its accompanying SN 2016jca with the GTC-OSIRIS, using grisms $\mathrm{R} 1000 \mathrm{~B}$ and $\mathrm{R} 1000 \mathrm{R}$. We obtained an additional spectrum of the AG-dominated OT with the X-Shooter (XS) instrument (Vernet et al. 2011) mounted on Unit Telescope 2 (UT2, Kueyen) of the Very Large Telescope (VLT) at the Paranal Observatory. We also present an optical spectrum obtained by the Public ESO Spectroscopic Survey of Transient Objects (PESSTO; Smartt et al. 2015) that used the EFOSC2 instrument mounted on ESO's $3.58 \mathrm{~m}$ New Technology Telescope (NTT), obtained on 04 January, 2017 (Chen et al. 2017). The GTC and NTT spectra were reduced using standard techniques with IRAFbased scripts, while the XS spectra were reduced using IRAF and IDL routines. Our spectroscopic observation log is found in Table C.1, and the spectroscopic time-series is presented in Fig. 6.

\section{High-energy emission}

\subsection{Gamma-rays}

GRB 161219B was observed in $\gamma$-rays by Swift-BAT, KonusWind and by the POLAR GRB polarimeter. We reduced the Swift-BAT data using the standard pipeline batgrbproduct, and then analysed the spectrum, integrated over the $T_{90}=6.9 \mathrm{~s}$ duration, with XSPEC (Arnaud 1996). The best-fitting model to the data is a single power-law (SPL) function with an exponential cutoff at the observed energy $E_{0}=92.3_{-29.0}^{+68.2} \mathrm{keV}$, and a powerlaw photon index of $\Gamma_{\gamma}=-1.40_{-0.24}^{+0.23}$, which are in agreement with similar analysis (Palmer et al. 2016). These values correspond to an intrinsic peak energy of $E_{\mathrm{p}, \mathrm{i}}=62.9_{-19.9}^{+47.0} \mathrm{keV}$ and a total isotropic energy emitted in the range $(1-10000 \mathrm{keV})$ of $E_{\gamma, \text { iso }}=8.50_{-3.75}^{+8.46} \times 10^{49} \mathrm{erg}$.

These quantities indicate that GRB 161219B is an outlier in the $E_{\mathrm{p}, \mathrm{i}}-E_{\gamma, \text { iso }}$ plane (i.e. the Amati relation; Amati et al. 2002). 3 All spectra presented in this paper are publically available at http:
//grbspec.iaa.es/ (de Ugarte Postigo et al. 2014a).

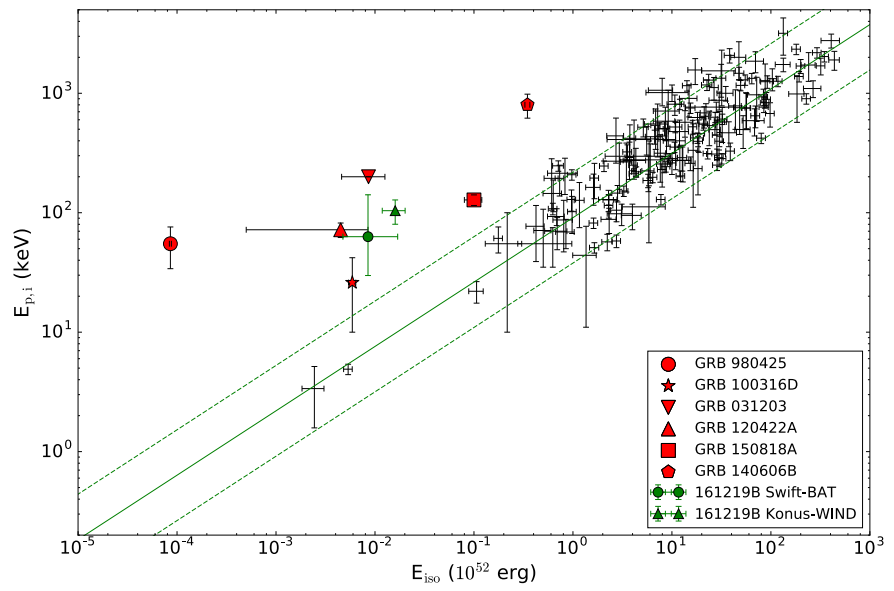

Fig. 2. Position of the intermediate-luminosity GRB 161219B in the $E_{\mathrm{p}, \mathrm{i}}-E_{\text {iso }}$ (Amati) plane. Shown for comparison is the GRB sample presented in Amati et al. (2002) and (Cano et al. 2015), as well as other outliers of the Amati relation, including low-luminosity GRBs 980425 , $031203 \& 100316 \mathrm{D}$, intermediate-luminosity GRB 150818A and highluminosity GRBs 120422A \& 140606B.

In Fig. 2 we have plotted for comparison low-luminosity GRBs (Cano et al. 2017; Martone et al. 2017), including GRB 980425 (Galama et al. 1998), GRB 031203 (Malesani et al. 2004) and GRB 100316D (Starling et al. 2011); intermediate-luminosity GRB 150818A (Palmer et al. 2015; Golenetskii et al. 2015), and high-luminosity GRBs 120422A (Schulze et al. 2014) and 140606B (Cano et al. 2015). We also fit the Konus-Wind data using an identical method, and again found that GRB 161219B is an outlier in the Amati relation.

In terms of its $\gamma$-ray luminosity, where $L_{\gamma \text {,iso }}=E_{\gamma \text {,iso }}(1+$ z) $t_{90}^{-1}$, we find $L_{\gamma}=\left(1.41_{-0.62}^{+1.41}\right) \times 10^{49} \mathrm{erg} \mathrm{s}^{-1}$, and $\log _{10}\left(L_{, \gamma}\right)=49.15_{-0.25}^{+0.30}$. Using the definitions given in the introduction, GRB $161219 \mathrm{~B}$ is an intermediate-luminosity GRB. Other examples of intermediate-luminosity GRBs include GRB 120714B (Cummings et al. 2012; Klose et al. 2012), GRB 130702A (D'Elia et al. 2015; Toy et al. 2016), and GRB 150818A (Palmer et al. 2015; Golenetskii et al. 2015; de Ugarte Postigo et al. 2015).

\subsection{X-rays}

We fit the Swift-XRT X-ray LC (see Sect. 4) with a broken power-law (BPL; Beuermann et al. 1999) to find the power-law decay indices $\alpha_{1}$ and $\alpha_{2}$, and the time the LC transitions $\left(t_{\mathrm{B}}\right)$ between them. Allowing all of the parameters to vary freely, our best-fitting results are: $\alpha_{1}=0.79 \pm 0.02, \alpha_{2}=1.93 \pm 0.28$, and $t_{\mathrm{B}}=38.0 \pm 7.3$ days $\left(\chi^{2} /\right.$ d.o.f. $\left.=389.2 / 348\right)$. Note that we excluded all data before $t-t_{0}=0.05$ days due to the presence of an early flare, which peaked at roughly $400 \mathrm{~s}$ after the first detection of the GRB. The data and best-fitting model are presented in Fig. 3.

The rest-frame break-time measured here ( $33.1 \pm 6.4$ days) is at a much later time than that determined by Ashall et al. (2017), who found a break-time of $\approx 12$ days $(\approx 13.8$ days observerframe), fit over a shorter time interval (up to +30 days). We note that if we force a break-time of $t_{\mathrm{B}}=14$ days, we obtain decay indices of $\alpha_{1}=0.72 \pm 0.01, \alpha_{2}=1.39 \pm 0.05$ $\left(\chi^{2} /\right.$ d.o.f. $\left.=394.6 / 349\right)$. 


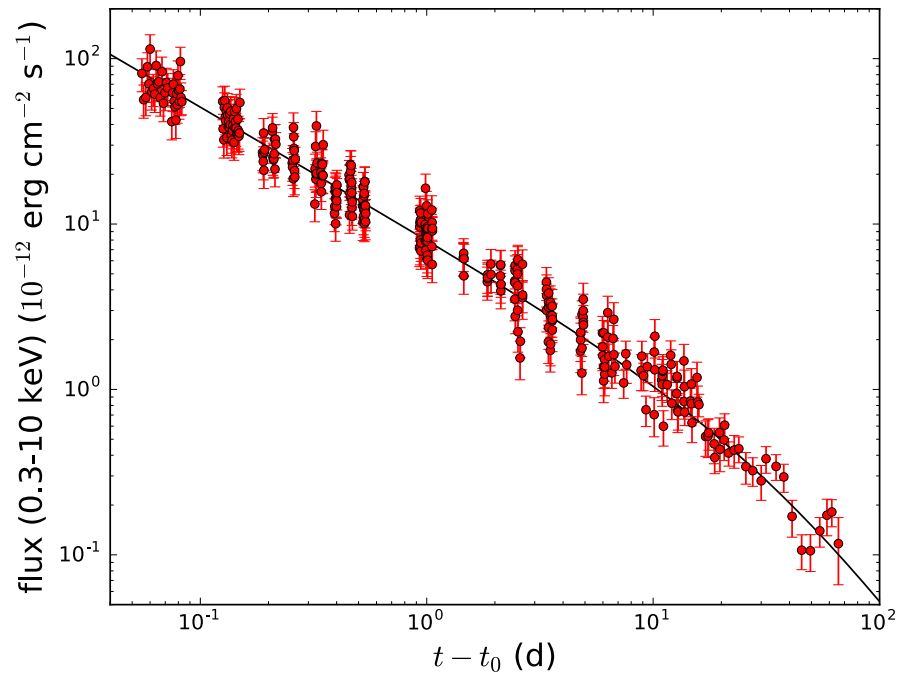

Fig. 3. Swift-XRT LC of GRB 161219B/SN 2016jca (orbit 1 was omitted - see the main text for more details). A broken power-law was fit to the data, with the best-fitting parameters being: $\alpha_{1}=0.79 \pm 0.02$, $\alpha_{2}=1.93 \pm 0.28$, and the time the LC breaks between $\alpha_{1}$ and $\alpha_{2}$ being $38.0 \pm 7.3$ days $\left(\chi^{2} /\right.$ d.o.f. $\left.=389.2 / 348\right)$.

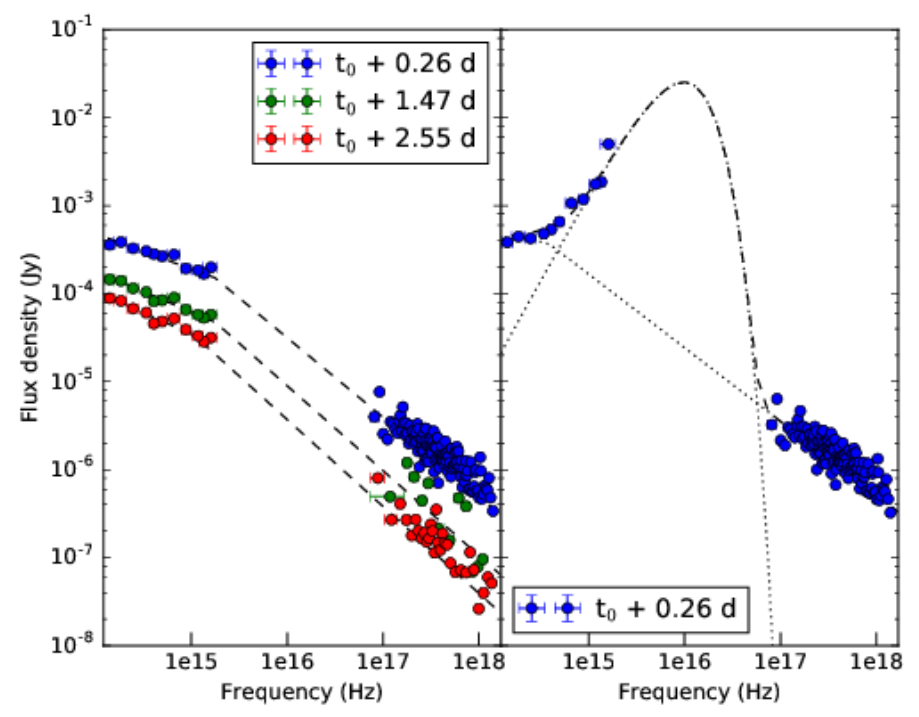

Fig. 4. NIR to X-ray SED of GRB $161219 \mathrm{~B}$ at $t-t_{0}=0.26,1.47$ and 2.55 days. Left: best-fitting model consisting of a broken power-law and MW extinction curve. For all three epochs, we find: $\beta_{\text {opt }} \approx 0.45$, $\beta_{\mathrm{X}} \approx 0.95$ and a break frequency of $\nu_{\mathrm{B}} \approx 1.7-1.8 \times 10^{15} \mathrm{~Hz}$. The local reddening is low $(E(B-V) \approx 0.02 \mathrm{mag})$. Right: to the SED at +0.26 days, we fit an extinguished broken power-law with an additional blackbody component. At this early epoch $(\approx+5.4 \mathrm{~h}$, rest-frame), the BB component is very hot $\left(T_{\mathrm{BB}} \approx 0.16 \times 10^{6} \mathrm{~K}\right)$ and it contributes $\approx 68 \%$ of the total recorded flux. (Note that the BB fit implies a larger local reddening than the BPL fits - see the main text for further discussion).

\section{The spectral energy distribution}

We modelled the spectral energy distribution (SED) from NIR to $\mathrm{X}$-ray frequencies in order to determine any dust extinction in the GRB's local environment. We calculated flux densities from the GROND (AB) magnitudes fluxes complemented by flux densities calculated from the Swift-UVOT AB magnitudes from optical to near-UV wavelengths, and Swift-XRT data. We computed SEDs at three different epochs, whose mean times were chosen with respect to Swift-XRT data: 1) $t-t_{0}=0.26$ days; 2) $t-t_{0}=1.47$ days; and 3) $t-t_{0}=2.55$ days. The XRT data were reduced with the standard XRTPIPELINE tool in caldb (version 20160609), after which we extracted time-resolved spectra that corresponded to the three different SED epochs using XSELECT. For the first SED, data suffered from pile-up and, therefore, we used an annular region with an inner radius of 5 pixels and an outer one of 30 pixels, while for the remaining two SEDs we used a circular region with a radius of 20 pixels.

UVOT flux densities were computed using standard SwiftUVOT data-reduction procedures (caldb version 20170130). First, we determined the regions for the source and background from the summed UVOT- $v$-band image, using circular apertures with a radius of $6^{\prime \prime}$ for both regions. Then we used the UVOTMAGHIST tool on the level 2 fits images to determine AB flux densities in each filter. Next, using the foreground-corrected flux densities of the $\mathrm{AG}+\mathrm{SN}+$ host determined from the GROND observations, we fit a SPL to the LCs and interpolated the flux densities to the time of the second and third SEDs. The flux densities for the first epoch, which occurred before the first GROND epoch (+0.286 days), were determined by extrapolating the SPL to the time of the first SED. All Swift-UVOT flux densities were determined via LC interpolation as the UVOT magnitudes were obtain before and after the times of the chosen SED epochs. Note that the UVOT magnitudes/fluxes are contaminated with host flux: as no pre-explosion UVOT templates of the GRB exist, we were unable to remove this component from the analysis. As such, in order to provide a consistent analysis, all fluxes modelled in this section have not had the host flux removed. The UVOT magnitudes, which are in the AB system, used in our analysis are presented in Table D.1.

The final NIR-to-Xray SEDs were fitted using XSPEC (Arnaud 1996). During the fit (following the general procedure described in Schady et al. 2010), we included two dust and gas components corresponding to the Galactic (using the UV/optical/NIR extinction law fom Cardelli et al. 1989) and host galaxy photoelectric absorption (Wilms et al. 2000) and dust extinction (using the MW/SMC/LMC templates from Pei 1992), where we fixed the Galactic values to $E(B-V)=0.028$ mag and $N(H)=3.06 \times 10^{20} \mathrm{~cm}^{-2}$ (Willingale et al. 2013). For the Galactic and host X-ray absorption component, we used the Tuebingen-Boulder ISM absorption model. For the fit, and in addition to the three dust extinction templates, we tried several different scenarios: (1) a SPL with no break between the X-ray and UV/optical/NIR regimes (e.g. Zafar et al. 2011); (2) a BPL, where the cooling break occurs between these regimes (i.e. $\left.\beta_{\mathrm{X}}=\beta_{\text {opt }}+0.5\right)$; (3) a BPL with the break frequency between the optical and X-ray, but we allowed the spectral indices to vary freely with no constraints.

We used the first epoch at $t-t_{0}=0.26$ days to determine which of these scenarios provided the best fit. First, the simple absorbed SPL function resulted in a goodness-of-fit of $\chi^{2} /$ d.o.f. $=662.6 / 573$, with a spectral index of $\beta=0.77 \pm 0.02$. Next, for scenario (2), we found spectral indices of $\beta_{\mathrm{opt}}=$ $0.40 \pm 0.07$ and $\beta_{\mathrm{X}}=0.90 \pm 0.07$, with a break frequency of $v_{\mathrm{B}}=(1.76 \pm 1.91) \times 10^{15} \mathrm{~Hz}\left(\chi^{2} /\right.$ d.o.f. $\left.=433.2 / 573\right)$, which is just outside of the UVOT frequency range (i.e. in the UV). The reddening was found to be $E(B-V)=0.026 \pm 0.020$ mag, with identical values found (within their respective errorbars) for all three dust extinction templates. For scenario (3), where the spectral indices were allowed to vary, we found similar values for the free parameters: $\beta_{\mathrm{opt}}=0.44 \pm 0.08$ and $\beta_{\mathrm{X}}=0.89 \pm 0.09$, with a break frequency of $v_{\mathrm{B}}=(1.71 \pm 1.92) \times 10^{15} \mathrm{~Hz}$, and an extinction of $E(B-V)=0.016 \pm 0.023 \mathrm{mag}\left(\chi^{2} /\right.$ d.o.f. $\left.=433.3 / 572\right)$. These results suggest that there is very little extinction local to the GRB. 
Although the $\chi^{2}$ statistic is more favourable for scenario (1), we ruled against this model for two reasons: first, when fitting the optical and X-ray regimes separately, we found very different spectral indices of $\beta_{\text {opt }}=1.10 \pm 0.07\left(\chi^{2} /\right.$ d.o.f. $\left.=8.1 / 7\right)$ and $\beta_{\mathrm{X}}=1.98 \pm 0.10\left(\chi^{2} /\right.$ d.o.f. $\left.=356.0 / 566\right)$. Secondly, an $F$-test between scenarios (1) and (3) show that the latter scenario (i.e. the BPL) is favoured: the $F$-value is $F=302.7$, with a probability of $9.9 \times 10^{-55}$. We therefore conclude that the NIR to X-ray SED at $t-t_{0}=0.26$ days is best-fit with an extinguished BPL, which is similar to the SED modelling results of Ashall et al. (2017).

For the other two epochs at $t-t_{0}=1.47$ days and 2.55 days we found similar results for all free parameters, with comparable values of the fit-goodness parameters for all three scenarios. The optical and X-ray indices do not vary much between $0.26-2.55$ days, which have an average value of $\beta_{\text {opt }} \approx 0.45$ and $\beta_{\mathrm{X}} \approx 0.95$, while the break frequency is approximately $v_{\mathrm{B}} \approx 1.7-1.8 \times 10^{15} \mathrm{~Hz}$. The error-bars on the break frequency are too large to determine if it increases or decreases with time. Finally, the weighted average of the line-of-sight host extinction is $E(B-V)_{\text {host,weighted }}=0.017 \pm 0.012 \mathrm{mag}$, which is the value used throughout this paper.

\section{An extra blackbody component?}

Motivated by previous studies that found an extra thermal component in the early X-ray spectra of many GRBs (e.g. Campana et al. 2006; Starling et al. 2011; Page et al. 2011; Thöne et al. 2011; Sparre \& Starling 2012; Friis \& Watson 2013; Schulze et al. 2014, we also performed a fourth fit where we included an extra blackbody (BB) component. For the first epoch, we found a $\mathrm{BB}$ component of temperature $T_{\mathrm{BB}} \approx 0.16 \times$ $10^{6} \mathrm{~K}$ and radius $R_{\mathrm{BB}} \approx 6 \times 10^{14} \mathrm{~cm}\left(\chi^{2} /\right.$ d.o.f. $\left.=404.3 / 578\right)$. An $F$-test between this model and scenario 3 gives an $F$-value of 20.44 and a probability of $2.7 \times 10^{-9}$, indicating it may provide a better fit to the data despite the increase in free parameters. In relative terms, the fit suggests that the BB component contributes $68 \%$ of the total flux at this epoch. We also find that the fit gives a larger rest-frame reddening of $E(B-V)=0.16 \pm 0.13 \mathrm{mag}$ than that determined from the reddened BPL model used in the previous section.

We fit the latter two epochs with the same fit. For the second epoch, we found that the fractional contribution of the $\mathrm{BB}$ component decreased to $\approx 10 \%$. The $\mathrm{BB}$ fit to the third epoch was entirely unconstrained. For the second epoch, we found the temperature decreased to $T_{\mathrm{BB}} \approx 32000 \mathrm{~K}$, while the $\mathrm{BB}$ radius was roughly the same as the first epoch. We note that the errorbars are too large to infer any changes/evolution. Again, the fit suggests larger host-extinction of $E(B-V) \approx 0.15 \mathrm{mag}$, but it is very poorly constrained.

The presence of a possible $\mathrm{BB}$ component is only convincing in the first epoch $\left(t-t_{0}=0.26\right.$ days), and entirely unconvincing in the third. As such, the suggestion of an extra BB component is tentative. However, if we take these results at face-value, the cooling thermal component could imply the presence of a thermal cocoon surrounding the jet, which is very hot early on, but fades rapidly, and does not contribute any appreciable flux after a couple days (rest-frame). However, the cocoon radius estimated for GRB 130925A by Piro et al. (2014) is of order $0.4-1.4 \times 10^{11} \mathrm{~cm}$, which is more than three orders of magnitude smaller than the radius found here. Alternatively, the thermal component could arise from a scenario similar to that suggested by Campana et al. (2006) for GRB 060218, where the shock breakout was trapped in an optically-thick stellar wind, breaking out only after the wind became optically thin. A consequence of this model are the pre-maximum peaks seen in the optical and UV LCs of GRB 060218, which are not seen here. We note that the alternative scenario presented by Margutti et al. (2015) and Nakar (2015), where the breakout occurs from a low-mass, extended envelope surrounding the progenitor, also predicts the achromatic pre-maximum bumps. Regardless, Campana et al. (2006) found that the BB radius of the thermal component evolved from $\approx 5 \times 10^{11} \mathrm{~cm}$ at $+300 \mathrm{~s}$ to $\approx 3 \times 10^{14} \mathrm{~cm}$ at 0.9 days. At +0.3 days it had a radius of $\approx 10^{13} \mathrm{~cm}$, almost two orders of magnitude smaller than that found here. Next, Starling et al. (2011) found a radius of $\approx 8 \times 10^{11} \mathrm{~cm}\left(t-t_{0}<800 \mathrm{~s}\right)$ for GRB $100316 \mathrm{D}$. For the sample of LGRBs presented in Starling et al. (2012), the BB radii determined from early-time X-ray spectra $\left(t-t_{0}=80-800 \mathrm{~s}\right)$, range from $0.03-9 \times 10^{12} \mathrm{~cm}$. A similar range of radii was determined by Page et al. (2011) for GRB 090618 for early-time $\mathrm{X}$-ray spectra.

Interestingly, a BB component was found in a fit of the X-ray to NIR SEDs of GRB 120422A by Schulze et al. (2014), where at +0.267 days, they find a BB temperature of $T_{\mathrm{BB}} \approx$ $0.19 \times 10^{6} \mathrm{~K}$, and a radius of $R_{\mathrm{BB}} \approx 7 \times 10^{13} \mathrm{~cm}$, which is about one order of magnitude smaller than that found here. This BB component was interpreted as thermal emission arising from the cooling stellar envelope following shock breakout, and a similar interpretation of the thermal component for GRB 161219B is appealing. Note that pre-maximum bumps were not observed for GRB 120422A, similar to GRB 161219B.

\section{The afterglow}

The observer-frame optical (griz) and NIR ( $J H K)$ LCs of GRB 161219B/SN 2016jca are shown in Figs. 5 and 7.

To quantify the three sources of flux portrayed in the LCs (the AG, the SN and the host galaxy, e.g. Zeh et al. 2004), we first de-reddened the observations of the OT for foreground extinction (see Sect. 4 and Table 1). We then converted the magnitudes into monochromatic flux densities using the $\mathrm{AB}$ zeropoint flux density (for griz) and the flux density zeropoints for $J H K$ from Greiner et al. (2008). The foreground de-reddened host flux densities in each optical filter were subtracted, resulting in LCs of just the optical transient (Fig. 5), which were then corrected for host/local extinction. Unfortunately, pre-explosion images of the host in the NIR filters $J H K$ do no exist (they are too shallow in the 2MASS survey), and hence the NIR data in Fig. 7 are de-reddened but not host subtracted.

In order to quantify the AG component we fit both a SPL and a BPL to the optical/NIR LCs. In this study, we did not assume that the AG behaves achromatically (e.g. Klose et al. 2004; Kann et al. 2016). In filters griz we also included a template $\mathrm{SN}$ in the fit (SN 1998bw), and simultaneously determined how much SN 2016jca was brighter/fainter $(k)$ and wider/slimmer $(s)$ than the template (see Sect. 7). The fitting was done using scripts written in pyxplot, as employed in previous works (Cano et al. 2011a,b, 2014b, 2015), which use linear-least squares fitting to find the best-fitting values of each one of these free parameters. Our results are presented in Table 2.

It can be seen that the assumption of achromatic AG behaviour in other studies would also be justified in this case. For all filters, a SPL provided the best fit to the optical observations, where in all filters the decay index was $\alpha \approx 0.8$ in griz and in the NIR it was $\alpha \approx 0.6$. In comparison, the decay index at times $<40$ days in the X-ray regime was found to be $\alpha_{\mathrm{X}}=0.79 \pm 0.02$, in excellent agreement with that found in the optical filters. One 

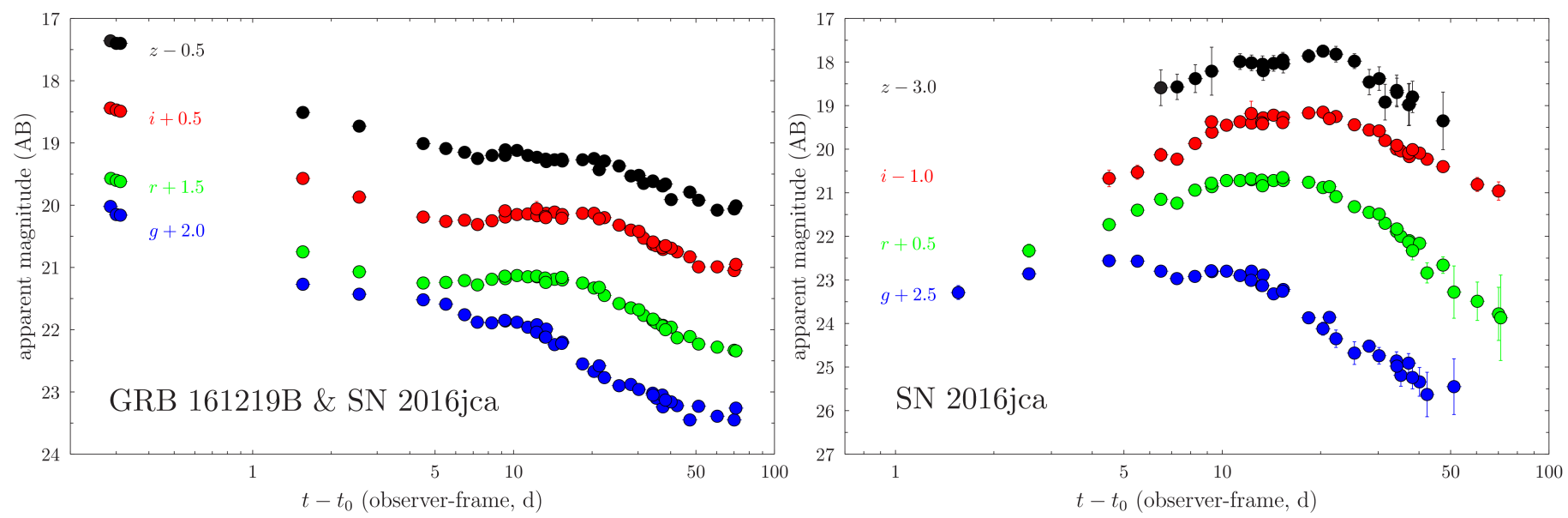

Fig. 5. Observer-frame optical (griz) LCs of GRB 161219B/SN 2016jca. Left: LCs of the AG, SN and underlying host, which are uncorrected for extinction. The typical evolution from the AG-dominated to the SN-dominated phase is seen in all optical filters. Right: host- and AG-subtracted LCs of SN 2016jca. A pre-maximum bump is seen in the $g$-band, which peaks around $\approx 5-6$ days. This bump is conspicuously absent in the other filters, and the origin of this flux excess is discussed in Sect. 9. In contrast, the potential bump seen in the $z$-band LC around 10 days is not real, but arises from instrumental defects.

Table 2. AG and SN phenomenological properties.

\begin{tabular}{cccccccc}
\hline \hline Band & $\alpha_{1}$ & $\alpha_{2}$ & $t_{\mathrm{B}}$ (day) & $k$ & $s$ & $m_{\mathrm{p}}(\mathrm{mag})$ & $t_{\mathrm{p}}(\mathrm{day})$ \\
\hline X-ray & $0.79 \pm 0.02$ & $1.93 \pm 0.28$ & $38.0 \pm 7.30$ & - & - & - & - \\
$\mathrm{X}$-ray & $0.72 \pm 0.01$ & $1.39 \pm 0.05$ & $14.0^{\dagger}$ & - & - & - & - \\
$g$ & $0.86 \pm 0.06$ & - & - & $0.77 \pm 0.08$ & $0.58 \pm 0.05$ & $20.31 \pm 0.05$ & $9.5 \pm 1.0$ \\
$r$ & $0.80 \pm 0.05$ & - & - & $0.78 \pm 0.09$ & $0.78 \pm 0.03$ & $20.18 \pm 0.05$ & $14.1 \pm 1.0$ \\
$i$ & $0.76 \pm 0.07$ & - & - & $0.81 \pm 0.06$ & $0.89 \pm 0.03$ & $20.20 \pm 0.04$ & $17.4 \pm 1.1$ \\
$z$ & $0.75 \pm 0.04$ & - & - & $0.51 \pm 0.06$ & $0.64 \pm 0.03$ & $20.90 \pm 0.05$ & $17.8 \pm 0.9$ \\
$J$ & $0.61 \pm 0.05$ & - & - & $0.83 \pm 0.12$ & $0.87 \pm 0.12$ & $19.54 \pm 0.10$ & $25.8 \pm 2.5$ \\
$H$ & $0.59 \pm 0.05$ & - & - & - & - & - & - \\
$K$ & $0.63 \pm 0.26$ & - & - & - & - & - & - \\
\hline$B$ & $0.76 \pm 0.04$ & - & - & $0.65 \pm 0.09$ & $0.57 \pm 0.12$ & $20.52 \pm 0.05$ & $7.9 \pm 1.3$ \\
$V$ & $0.79 \pm 0.06$ & - & - & $0.79 \pm 0.09$ & $0.79 \pm 0.11$ & $20.18 \pm 0.05$ & $12.3 \pm 0.7$ \\
$R$ & $0.76 \pm 0.04$ & - & - & $0.80 \pm 0.12$ & $0.89 \pm 0.09$ & $20.02 \pm 0.06$ & $15.8 \pm 1.7$ \\
\hline
\end{tabular}

Notes. $\dagger$ Break-time fixed during fit. NB: X-ray data and filters grizJHK are for observer-frame filters and times. Rest-frame properties are given for $B V R$. Filters griz are in the $\mathrm{AB}$ system, while $B V R$ and $J H K$ are in the Vega system. Properties in filters griz and $B V R$ have been host-subtracted, whereas those in $J H K$ are not.

caveat to the fitting is that the host-contribution was only removed from the optical observations and not the NIR, where pre-explosion imaging in $J H K$ is not available. The effect of having the host flux in the NIR LCs is that the decay rate will be slower than in reality: as the AG fades, the host contributes an increasing portion of flux to the LC. Thus the AG will (incorrectly) appear to fade at a slower rate, and hence have a smaller value of $\alpha$.

\section{SN 2016jca: observational properties}

\subsection{Photometric properties}

As well as determining the AG behaviour in Sect. 6, we simultaneously fit the AG and SN to determine the luminosity, $k$, and stretch, $s$, factors of SN 2016jca relative to SN 1998bw. The luminosity factor is similar in optical filters gri, with a value of $k \approx 0.8$, but it is fainter in the $z$-band $(k=0.5)$. The stretch factors in filters $g$ and $z$ are $s \approx 0.6$, while in riJ they are $s=0.8-$ 0.9. Collectively, these results show that in all filters gri, the SN is fainter and evolves more quickly than SN 1998bw.
We also fit the AG- and host-subtracted SN LCs with two models: (1) a model based on the equations in Bazin et al. (2011); and (2) high-order polynomials, in order to determine the time and magnitude of maximum light in each filter. These are also given in Table 2. It is seen that the $\mathrm{SN}$ peaks later in redder filters, as expected.

Finally, we fit the rest-frame SN LCs (i.e. K-corrected; see Sect. 7.3) in $B V R$ to determine their peak magnitudes and time of peak light. As with the observer-frame filters, the SN peaks at later times in redder filters. Relative to SN 1998bw, SN 2016jca was fainter $(k=0.65-0.80)$, and reached peak light before SN 1998bw ( $s=0.57-0.89)$ in all filters.

Next, and using a distance modulus of $\mu=39.22 \mathrm{mag}$, we find rest-frame, peak absolute magnitudes of $M_{B}=-18.70 \pm$ $0.05, M_{V}=-19.04 \pm 0.05$ and $M_{R}=-19.20 \pm 0.06$. For comparison, Li \& Hjorth (2014) found for SN 1998bw a peak $V$ band absolute magnitude of $M_{V}=-19.3$. Thus SN 2016jca is roughly 0.25 mag fainter than the archetype GRB-SN 1998bw, which agrees with the fact that the luminosity factor in this filter $\left(k_{V}=0.79\right)$ is less than one. Relative to the rest-frame magnitudes of the GRB-SN sample in Li \& Hjorth (2014), SN 2016jca 


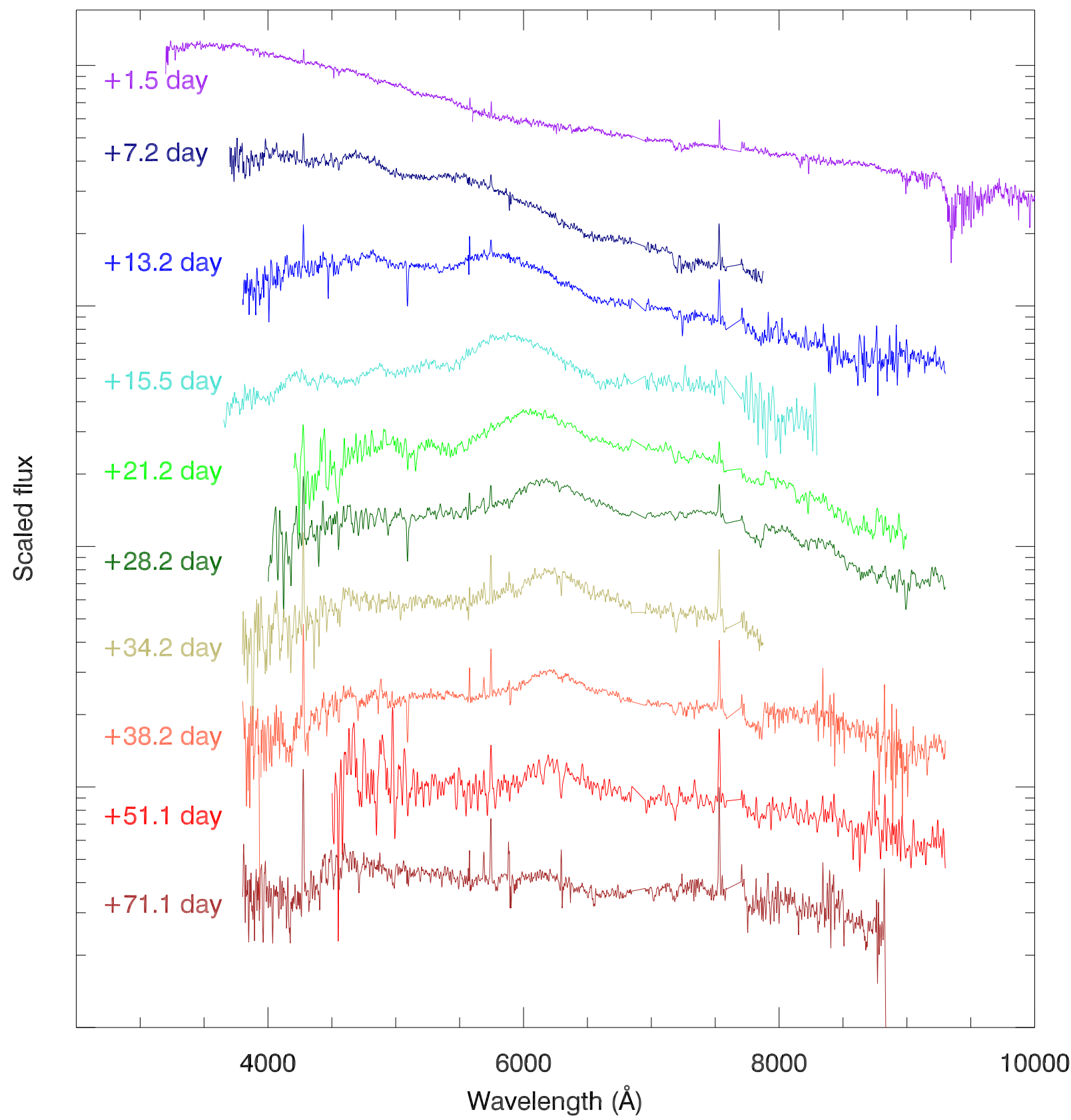

Fig. 6. Spectroscopic time-series of GRB 161219B \& SN 2016jca. Wavelengths and times are presented in the observer-frame $(z=0.1475)$, and are not host-subtracted. Narrow emission lines are seen, which arise from star-forming regions within the host galaxy.

is quite faint, and is only brighter than SN 2006aj $\left(M_{V}=-18.85\right)$ and SN 2010bh $\left(M_{V}=-18.89\right)$.

\subsection{Spectroscopic properties}

Our spectroscopic time-series of GRB 161219B/SN 2016jca is shown in Fig. 6. The transition from the AG-dominated to the $\mathrm{SN}$-dominated phase is clearly portrayed in the shape and evolution of the optical spectra. The XS spectrum taken at $t-t_{0}=$ 1.5 days (1.3 days rest-frame) is flat and featureless, typical of GRB afterglow spectra (e.g. Fynbo et al. 2009). Host emission lines are superimposed upon the AG spectrum. The next spectrum, obtained at $t-t_{0}=7.5$ days (6.3 days rest-frame) shows unambiguous spectral features of a broad-lined SN, while the absence of both hydrogen and helium indicates a spectral class of type Ic. The broad absorption feature seen at observerframe $5200 \AA$ is attributed to blue-shifted Fe II $\lambda$ 15169, though other transitions may also be blended in. Near and after peak light, an additional absorption feature is seen near observerframe $6500 \AA$, which may be blueshifted Si II 26355 . A hint of blueshifted O I and/or Ca II near observer-frame $9000 \AA$ is seen in the GTC spectrum taken on 16-January-2017 $\left(t-t_{0}=\right.$ 28.2 days, observer-frame). Sky lines in the 22-January-2017 spectrum $\left(t-t_{0}=34.2\right.$ days, observer-frame $)$ inhibit our ability to detect the same feature.

A comparison of our GTC spectrum taken near peak $V$ band light (01 January, 2017) with other GRB-SNe near peak $V$-band light is shown in Fig. 8. The broad spectral features seen for SN 2016jca are quite typical of other GRB-SNe. In Fig. 9 we plotted the blueshifted velocities of Fe II $\lambda 15169$, under 
Z. Cano et al.: GRB 161219B/SN 2016jca: A low-redshift gamma-ray burst supernova powered by radioactive heating

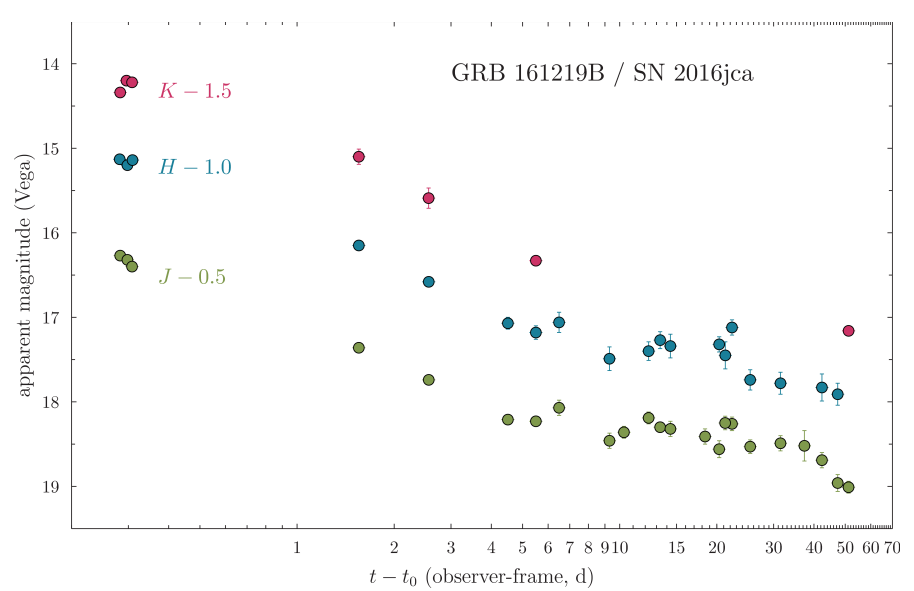

Fig. 7. Observer-frame NIR ( $J H K$ ) LCs of GRB 161219B/SN 2016jca. The magnitudes are not corrected for extinction, and they have an unknown contribution from the underlying host.

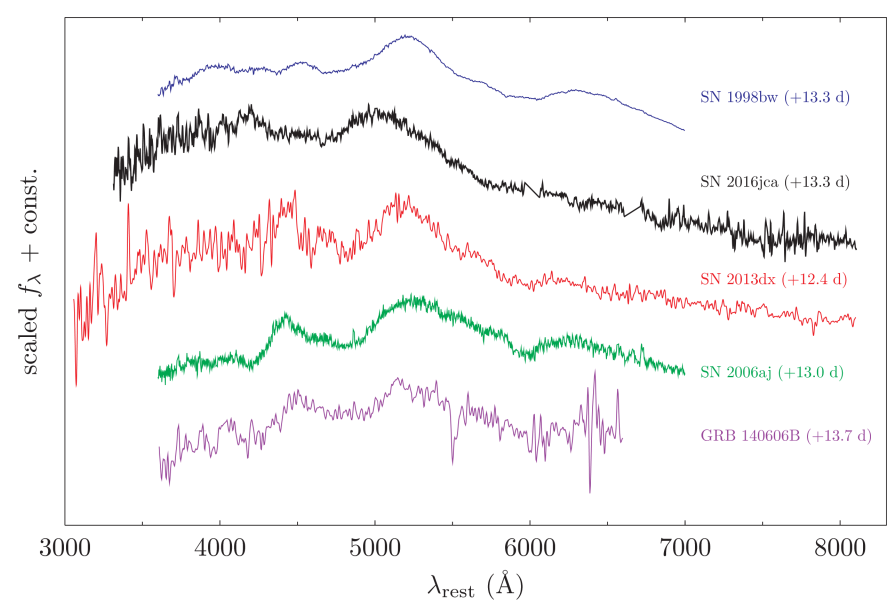

Fig. 8. Comparison of the spectrum of SN 2016jca obtained with the GTC on 01-January-2017 ( $t-t_{0}=13.3$ days; shown in black), which was obtained near peak $V$-band light $(+12.5 \mathrm{~d}$, rest-frame). Plotted for comparison is a sample of GRB-SNe at similar post-explosion times: SN 1998bw (+13.3 days; blue), SN 2006aj (+13.0 days; green), SN 2013dx (+12.4 days; red) and the SN associated with GRB 140606B $(+13.7$ days; purple). All times and wavelengths are shown in the restframe. Visual inspection of the spectra reveals that the trough bluewards of the peak around $5000 \sim 5200 \AA$, which we attribute to blueshifted Fe II $\lambda 5169$, occurs at bluer wavelengths for SN 2016jca than all the comparison GRB-SNe, thus highlighting its high-velocity nature.

the assumption that the absorption feature at observer-frame $5200 \AA$ is unblended with other transitions. It is seen that the magnitude and evolution of the Fe II $\lambda 5169$ is quite typical of other GRB-SNe. At peak bolometric light $\left(t-t_{0}=10.7\right.$ days, rest-frame; see Sect. 8), the line velocity is $v_{\mathrm{Fe}}=29700 \pm$ $1500 \mathrm{~km} \mathrm{~s}^{-1}$. As there were no data at the precise time of peak bolometric light, we determined the peak velocity by fitting a log-linear spline to the line velocity data, and extracted the velocity at the time of peak light. In comparison, Ashall et al. (2017) found a peak photospheric velocity of $\approx 26000 \mathrm{~km} \mathrm{~s}^{-1}$ from their spectral modelling. While good agreement is seen between the two analyses, we must consider the limitations of using a single transition as a proxy for the photospheric velocity (e.g. Modjaz et al. 2016).

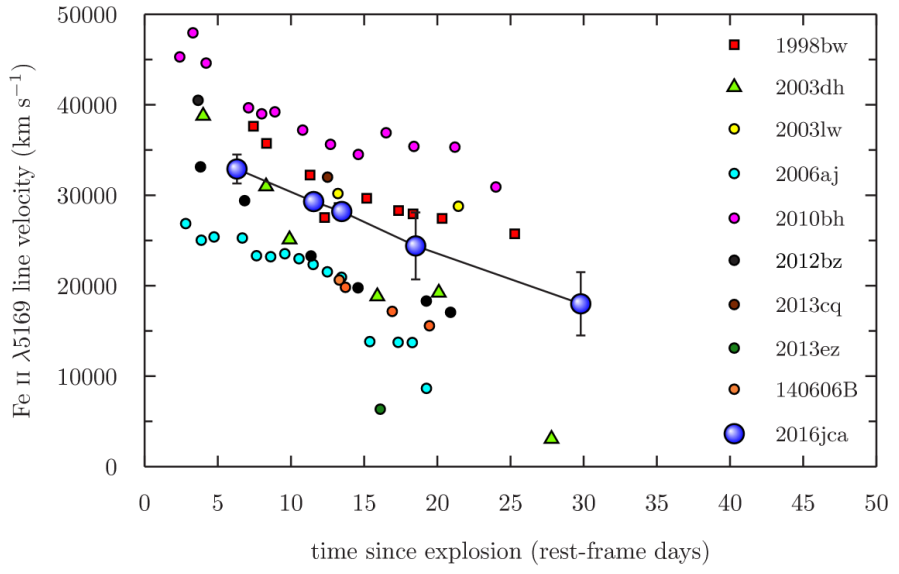

Fig. 9. Blue-shifted velocities of the Fe II $\lambda 5169$ transition.

\subsection{Luminosity-stretch relationship}

In an identical analysis as presented in Cano (2014), we investigated the rest-frame stretch $(s)$ and luminosity $(k)$ factors of SN 2016jca. The detailed procedure is described in Cano (2014), but briefly the main steps include: (1) create host-subtracted and de-reddened observer-frame SEDs in filters griz for each contemporaneous epoch. Then, using a redshift of $z=0.1475$, we interpolated to the precise red-shifted rest-frame wavelength in filters $B V R$, using the effective wavelengths from Fukugita et al. (1995). As such, our K-correction is obtained via SED interpolation. We then extracted the SED-interpolated flux at the desired red-shifted wavelength, recreating a LC similar to that shown in Fig. 5. Next, a SPL and template SN LC were simultaneously fit to the observations to obtain the decay index $\alpha$, as well as $s$ and $k$. The rest-frame properties are presented in Table 2 . It is seen that the decay index matches very well with those obtained from modelling the observer-frame filters.

We fit a straight line to the $k-s$ values $(N=24$, and d.o.f. $=$ 24-2 =22), finding a slope of $m=1.53 \pm 0.18$ and $y$-intercept $c=-0.26 \pm 0.13$. The errors were determined using a bootstrap method with Monte-Carlo sampling ( $N=10000$ simulations). The Pearson's correlation coefficient is $r=0.927$ and the twopoint probability of a chance correlation is $p=2.0 \times 10^{-10}$. The value of $r$ is well in excess of the critical value for 22 d.o.f. at the $p=0.001$ level. It is seen that the $k$ and $s$ values in $R$ are marginally consistent with the best-fitting line to within $1 \sigma$, while those in $B$ and $V$ are entirely consistent with the relationship. As discussed in Cano (2014), this statistically significant $k$-s (i.e. luminosity-stretch) relationship indicates that GRB-SNe have the potential to be used as standardizable candles (Cano et al. 2014c; Li \& Hjorth 2014) in SN-cosmology.

\section{SN 2016jca - bolometric properties}

We constructed a quasi-bolometric LC from our optical observations (de-reddened, host- and AG-subtracted) in griz. We followed the procedure outlined in detail in Cano et al. (2014a), which briefly, includes creating SEDs for each epoch, fitting a linear spline to the data, and integrating the spline between the frequency limits of the reddest and bluest filters (i.e. assuming no flux beyond these limits). We used the effective wavelengths given in Fukugita et al. (1995). Then, the flux bolometric LC was transformed to a luminosity bolometric LC using a distance of $700 \mathrm{Mpc}$. We estimated the luminosity errors by taking the average fractional uncertainty in each griz SED (which includes the 


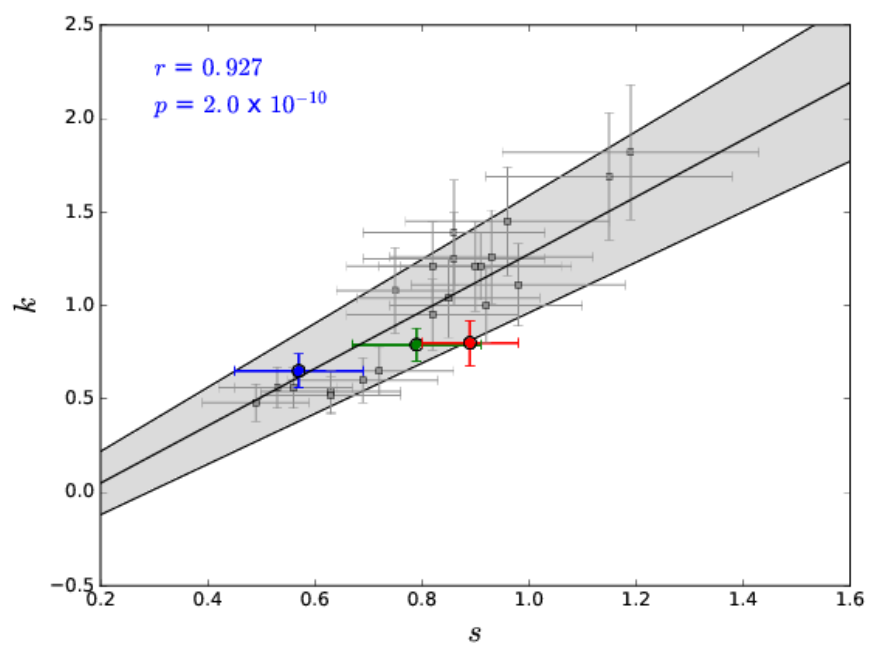

Fig. 10. Luminosity $(k)$-stretch $(s)$ relationship from Cano (2014). Plotted also are the rest-frame $s$ and $k$ values of SN 2016jca in $B$ (blue), $V$ (green) and $R$ (red). The best-fitting is shown in black, and the $1 \sigma$ error region is shown in shaded grey. The best-fitting values for the fitted line are found in the main text. The $k$ and $s$ values in $R$ are marginally consistent within $1 \sigma$, while those in $B$ and $V$ are entirely consistent with the relationship.

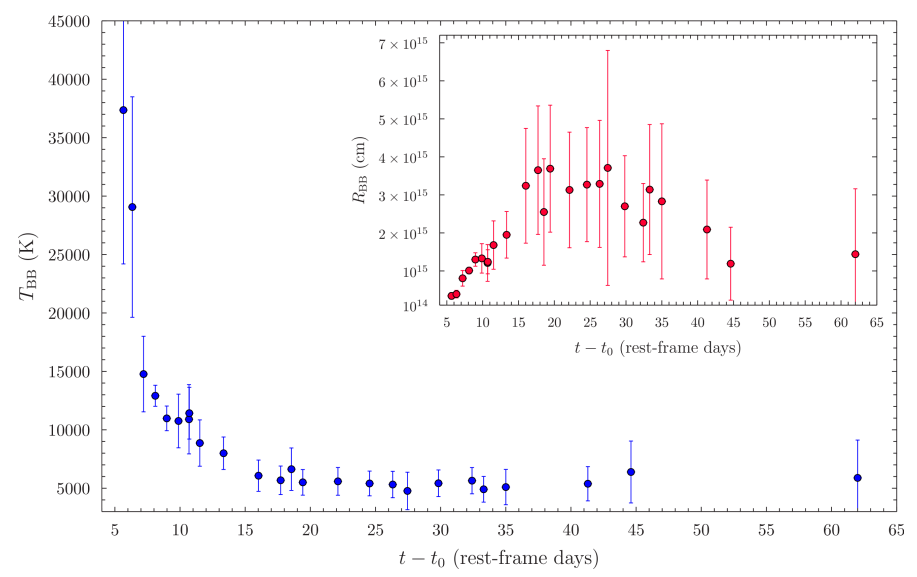

Fig. 11. Evolution of the $\mathrm{BB}$ colour temperature $\left(T_{\mathrm{BB}}\right.$, blue $)$ and radius (inset, red, $R_{\mathrm{BB}}$ ). The colour temperature corresponds to filters griz.

uncertainties in the photometry, AG model, and host photometry, all added in quadrature and propagated through the analysis), and applied this to the bolometric luminosity error. The final bolometric LC is shown in Fig. 12. The peak griz luminosity is $L=4.6 \times 10^{42} \mathrm{erg} \mathrm{s}^{-1}$, which occurs around +10.7 days (rest-frame).

\subsection{Temperature evolution}

Under the assumption that SN 2016jca emits as a pure BB, and does not suffer any dilution effects (which is likely to be an over-simplification of reality, e.g. Dessart \& Hillier 2005; Dessart et al. 2015), we fit the griz SEDs with a Planck function to determine the $\mathrm{BB}$ colour temperature $\left(T_{\mathrm{BB}}\right.$, in filters griz) and the radius $\left(R_{\mathrm{BB}}\right)$ of the $\mathrm{BB}$ emitter. Their evolution is plotted in Fig. 11.

From the figure, the BB temperature has an initial value of $T_{\mathrm{BB}}=37000 \mathrm{~K}$ at $t-t_{0}=5.6 \mathrm{~d}$, which then decreases rapidly, reaching a plateau of $T_{\mathrm{BB}} \approx 5000-6000 \mathrm{~K}$ after approximately 20 days. The $\mathrm{BB}$ radius is $\approx 3 \times 10^{14} \mathrm{~cm}$ during the first epoch, and reaches a maximum radius of $R_{\mathrm{BB}} \approx 3-4 \times 10^{15} \mathrm{~cm}$ around $t-t_{0}=20-30 \mathrm{~d}$, before decreasing to $R_{\mathrm{BB}} \approx 1-2 \times 10^{15} \mathrm{~cm}$ after 40 days.

\subsection{The radioactive heating model}

Currently, the accepted physical processes that are thought to power GRB-SNe are heating arising from the interaction of $\gamma$-ray photons emitted during the decay process of nickel and cobalt into their daughter products (i.e. the radioactive heating model, Arnett 1982), and energy input from a magnetar central engine, whose presence has been invoked for SLSNe-I and the very luminous GRB-SN $2011 \mathrm{kl}$ (Greiner et al. 2015). The first model is considered in this section, while the latter in the following subsection.

Two versions of the radioactive heating model (see Appendix A) were fit to the griz bolometric LC of SN 2016jca: one that assumes that all emitted $\gamma$-rays are thermalised in the $\mathrm{SN}$ ejecta (red model in Fig. 12), and another that allows a fraction of the $\gamma$-rays to escape into space without interacting with, or depositing energy into the SN (black model in Fig. 12). In the latter model, an additional free-parameter is the $\gamma$-ray opacity $\left(\kappa_{\gamma}\right)$. Both models assume a grey optical opacity of $\kappa=0.07 \mathrm{~cm}^{2} \mathrm{~g}^{-1}$, and a peak photospheric velocity, as inferred from the Fe II $\lambda 5169$ line velocities, of $v_{\mathrm{ph}}=29700 \pm 1500 \mathrm{~km} \mathrm{~s}^{-1}$.

First, when the model that assumed full trapping of all emitted $\gamma$-rays (red line) was fit to the data, we find a nickel mass of $M_{\mathrm{Ni}}=0.22 \pm 0.08 M_{\odot}$, an ejecta mass of $M_{\mathrm{ej}}=5.8 \pm 0.3 M_{\odot}$, and a kinetic energy of $E_{\mathrm{K}}=5.1 \pm 0.8 \times 10^{52} \mathrm{erg}$.

Next, we fit the partial-trapping model (black) to the bolometric LC, finding a nickel mass of $M_{\mathrm{Ni}}=0.22 \pm 0.08 M_{\odot}$, an ejecta mass of $M_{\mathrm{ej}}=5.9 \pm 0.3 M_{\odot}$, and a kinetic energy of $E_{\mathrm{K}}=$ $5.2 \pm 0.8 \times 10^{52} \mathrm{erg}$. The bolometric properties obtained from both models agree very well, where the only difference is the slightly increased ejecta mass constrained by the partial-trapping model. We also found a $\gamma$-ray opacity of $\kappa_{\gamma}=0.034 \mathrm{~cm}^{2} \mathrm{~g}^{-1}$. To put the value of the $\gamma$-opacity into context, Wheeler et al. (2015) determined this value for a sample of $N=20 \mathrm{SNe}$ Ibc, finding $\kappa_{\gamma}=0.010 \mathrm{~cm}^{2} \mathrm{~g}^{-1}$ for the one GRB-SN in their sample (SN 1998bw), which is roughly three times smaller than that found here for SN 2016jca. Overall, Wheeler et al. (2015) find a range of $\gamma$-ray opacities $0.001 \leq \kappa_{\gamma} \leq 0.047$, for which SN 2016jca falls within the upper end of this range.

For SN 2016jca, Ashall et al. (2017) ${ }^{4}$ found a nickel mass of $M_{\mathrm{Ni}}=0.4 M_{\odot}$, an ejecta mass of $M_{\mathrm{ej}}=8 M_{\odot}$, and a kinetic energy of $E_{\mathrm{K}}=5.4 \times 10^{52} \mathrm{erg}$. These results were obtained from modelling both a bolometric LC (obtained over a wavelength range of 3000-10000 $\AA$ ) and radiative transfer modelling of their spectral time series. The results of each study are loosely consistent, though both the ejecta mass and nickel content therein found here are smaller than those found by Ashall et al. (2017). It has been shown in previous studies (Modjaz et al. 2009; Cano et al. 2011b; Lyman et al. 2014), that including data over different frequency ranges affects the constructed bolometric LC differently. Including bluer data causes the bolometric LC to peak earlier, while including NIR observation causes the LC to become wider and peak later. Importantly, the inclusion of more data clearly makes the bolometric LC brighter and more luminous. The wavelength range investigated here is 4900-9200 $⿱$,

\footnotetext{
4 At the time of publication, the Ashall et al. (2017) was still in archive format, and it is anticipated that their results will be amended in its final, published form. Hence, the discussion presented here is relative to their (preliminary) results.
} 

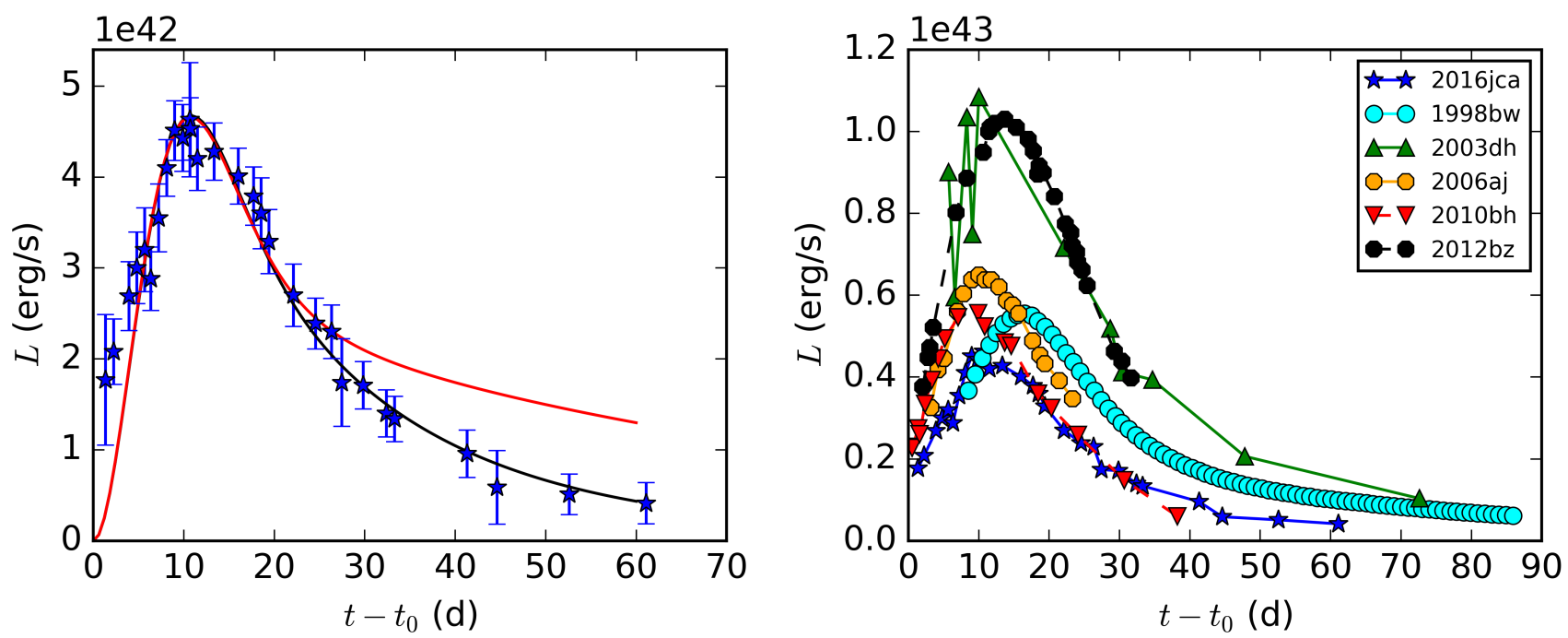

Fig. 12. Radioactive heating model (Arnett 1982) fit to the bolometric (griz) LC of SN 2016jca. Left: two versions of the model have been fit to the data, one that assumes full trapping of $\gamma$-rays (red), and one which allows $\gamma$-ray leakage (black). It is seen that the latter model better fits the data. Both models require a nickel mass of $M_{\mathrm{Ni}}=0.22 M_{\odot}$, an ejecta mass of $M_{\mathrm{ej}}=5.8 M_{\odot}$, and for a peak photospheric velocity of $v_{\text {ph }}=29700 \pm 1500 \mathrm{~km} \mathrm{~s}^{-1}$, a kinetic energy of $E_{\mathrm{K}}=5.1 \pm 0.8 \times 10^{52} \mathrm{erg}$. Both models assume a grey optical opacity of $\kappa=0.07 \mathrm{~cm}^{2} \mathrm{~g}^{-1}$. In the latter model, we find a $\gamma$-ray opacity of $\kappa_{\gamma}=0.034 \mathrm{~cm}^{2} \mathrm{~g}^{-1}$. Right: comparison of the optical bolometric LCs of a small sample of GRB-SNe: SN 1998bw (BVRI), SN 2003dh (UBVR), SN 2006aj (BVRI), SN 2010bh (griz) and SN 2012bz (griz).

which is smaller than that in Ashall et al. (2017), is clearly responsible for us finding smaller nickel and ejecta masses. Finally, given the similar peak photospheric velocities considered in each paper $\left(v_{\mathrm{ph}} \approx 26000 \mathrm{~km} \mathrm{~s}^{-1}\right.$, their Fig. 5), it is expected that we find similar explosion energies.

When visually comparing the bolometric LC of SN 2016jca to other GRB-SNe, it is seen that in relative terms, SN 2016jca is the least luminous. One caveat however is that while an attempt has been made to compare bolometric LCs of the GRB-SNe over similar wavelength ranges, this is not always possible. For example, the bolometric LC SN 2003dh was constructed from $U B V R$ observations (Deng et al. 2005), SN 1998bw is from $B V R I$ (Patat et al. 2001), SN 2006aj is from BVRI (Pian et al. 2006), SN 2010bh is from griz (Olivares et al. 2012) and SN 2012bz is from griz (Schulze et al. 2014). Next, relative to the "average" GRB-SNe (Cano et al. 2017), which has $M_{\mathrm{Ni}}=0.4 M_{\odot}(\sigma=$ $\left.0.2 M_{\odot}\right)$, an ejecta mass of $M_{\mathrm{ej}}=6 M_{\odot}\left(\sigma=4 M_{\odot}\right)$, and a kinetic energy of $E_{\mathrm{K}}=2.5 \times 10^{52} \mathrm{erg}\left(\sigma=1.8 \times 10^{52} \mathrm{erg}\right)$, SN 2016jca synthesised less radioactive material, but had a "typical" mass of ejecta. SN 2016jca is more energetic than the average GRB-SNe because its peak photospheric velocity is more rapid than that of the average GRB-SN $\left(v_{\mathrm{ph}}=20000 \mathrm{~km} \mathrm{~s}^{-1}\left(\sigma=8000 \mathrm{~km} \mathrm{~s}^{-1}\right)\right.$ by more than $1 \sigma$. As noted in other works (Mazzali et al. 2014; Ashall et al. 2017), the kinetic energies and ejecta masses determined from 1D analytical modelling should be considered as upper limits to their true values as they do not consider the true aspherical nature of the ejecta (e.g. Mazzali et al. 2001; Maeda et al. 2002, 2006; Wang \& Wheeler 2008).

\subsection{The magnetar model}

Next, we fit both the optical and X-ray data to see if the luminosity could plausibly be explained within the context of the magnetar model (see Appendix B), using the model from Cano et al. (2016). For the magnetar model to be deemed viable, the initial spin period $\left(P_{0}\right)$ and magnetic field strength $\left(B_{0}\right)$ of the magnetar central engine should be consistent when fitting the X-ray and optical data independently, otherwise the model is rejected.

First, we fit the Swift-XRT (top panel in Fig. 13). When fitting the SPL and magnetar-powered AG phases, the free parameters are the normalisation constant $(\Lambda)$ of the former, and the luminosity $\left(L_{0}\right)$ and duration $\left(T_{\mathrm{o}}\right)$ of the latter phases. The SPL index was fixed to $\alpha=\Gamma_{\gamma}+1=2.4$. The best-fitting model gives $L_{0}=(2.25 \pm 0.08) \times 10^{45} \mathrm{erg} \mathrm{s}^{-1}$ and $T_{0}=(1.39 \pm 0.06) \times 10^{5} \mathrm{~s}$. In turn this implies $B_{0}=(1.0 \pm 0.1) \times 10^{15} \mathrm{G}$, and $P_{0}=8.1 \pm 0.3 \mathrm{~ms}$. Visual inspection of the LC (top panel in Fig. 13) shows that the model is a poor fit to the data, where the observations after $t-t_{0}=3 \times 10^{5} \mathrm{~s}$ (rest-frame) fade at a slower rate than that of the magnetar model $\left(t^{-2}\right)$.

Next, we fit the magnetar model to an $r$-band luminosity LC (observer-frame griz) of GRB 161219B/SN 2016jca (bottom panel of Fig. 13), using an identical approach as that used in Cano et al. (2016). The free parameters in this model, in addition to $L_{0}$ and $T_{0}$, are the diffusion timescale $\left(t_{\text {diff }}\right)$ and a multiplicative factor $(\Psi)$, which is needed in cases where the luminosity of the magnetar-powered SN, as determined from the magnetar-powered AG phase, is under-luminous relative to the observations. The best-fitting model gives $L_{0}=(3.73 \pm$ $0.26) \times 10^{43} \mathrm{erg} \mathrm{s}^{-1}, T_{0}=(4.43 \pm 0.51) \times 10^{5} \mathrm{~s}, t_{\text {diff }}=9.43 \pm$ 0.10 days, and $\Psi=4.2 \pm 0.1$. In turn this implies $B_{0}=(2.4 \pm$ $0.3) \times 10^{15} \mathrm{G}$, and $P_{0}=35.2 \pm 3.0 \mathrm{~ms}$. As before for the X-ray LC, the late-time decay of the observations clearly deviates from that of the magnetar model.

The clear mismatch between the values determined from fitting the X-ray and optical data independently imply that the magnetar model cannot satisfactorily, and self-consistently, describe all phases of GRB 161219B/SN 2016jca. In the (unlikely) scenario that SN 2016jca is powered in part by emission arising from a magnetar central engine, an additional source of energy is needed to explain its $r$-band luminosity, which is likely to be radioactive nickel. In this case, an additional mass of $M_{\mathrm{Ni}} \sim 0.4 M_{\odot}$ is required. (Note that the required nickel mass in the magnetar model is larger than that inferred from modelling of the bolometric LC with the radioactive-heating model. In the 
GRB 161219B / SN 2016jca
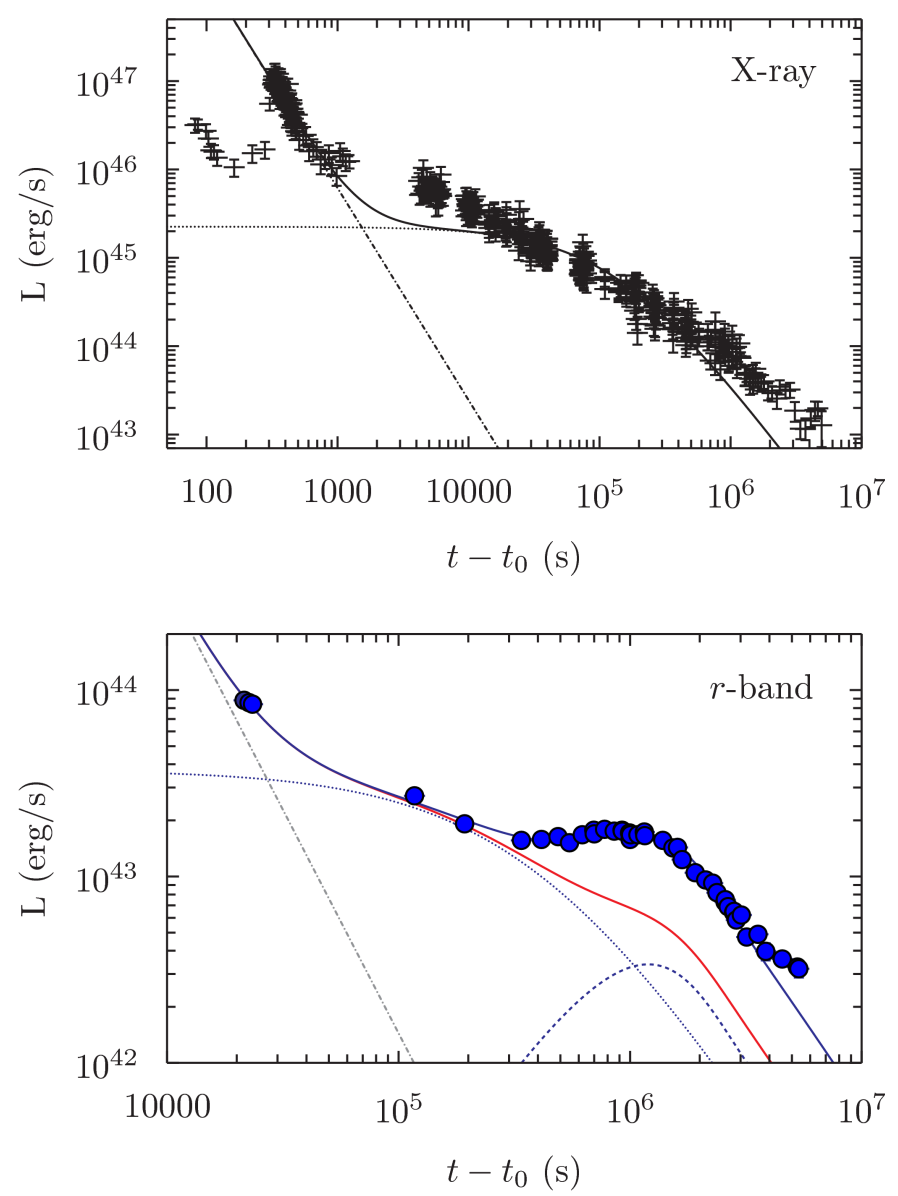

Fig. 13. Magnetar model from Cano et al. (2016) fit to the X-ray (top) and $r$-band (bottom) luminosity LCs. Times are shown in the restframe. Top: sum of the SPL (dot-dashed line) and magnetar-powered AG (dotted line) phases is shown as the solid black line. Visual inspection shows that the model fails to reproduce the decay seen in the observations at $t-t_{0}>3 \times 10^{5} \mathrm{~s}$. The best-fitting model gives $B_{0}=(1.0 \pm 0.1) \times 10^{15} \mathrm{G}$, and $P_{0}=8.1 \pm 0.3 \mathrm{~ms}$. Bottom: sum of the SPL (dot-dashed line), magnetar-powered AG (dotted line) and magnetarpowered SN (dashed line) phases is shown as the solid red line. The solid blue line includes an additional multiplicative factor $(\Psi)$ to get the magnetar-powered $\mathrm{SN}$ to match the observations. The best-fitting model gives $B_{0}=(2.4 \pm 0.3) \times 10^{15} \mathrm{G}$, and $P_{0}=35.2 \pm 3.0 \mathrm{~ms}$, which are clearly discrepant with those determined from the $\mathrm{X}$-ray. In addition, we found a diffusion time scale of $t_{\text {diff }}=9.43 \pm 0.10$ days, and $\Psi=4.2 \pm 0.1$. The conclusion is that the magnetar model cannot self-consistently explain the X-ray and optical observations of GRB 161219B/SN 2016jca. Even if SN 2016jca was powered in part by a magnetar central engine, an additional source of energy is needed to reproduce the observations, most likely a reservoir of radioactive nickel. The required nickel mass to get the model to match observations is $M_{\mathrm{Ni}} \sim 0.4 M_{\odot}$.

magnetar model, we are fitting the AG and SN simultaneously, and the AG decays as $t^{-2}$. When modelling the AG in Sect. 6, the AG is seen to decay as $t^{-0.8}$, thus the AG decays slower and contributes more flux to the later SN phase (which is then removed) than in the magnetar model. Hence, the bolometric LC constructed from the AG-subtracted data is fainter, and requires less nickel to explain its luminosity.)

We note that if the Fe II $\lambda 5169$ line velocities are a suitable proxy for the photospheric velocity, their evolution is inconsistent with that of the 1D magnetar model (e.g. Kasen \& Bildsten 2010; Wang et al. 2017; see Cano et al. 2017 for further discussion), which predicts a flat evolution. Inspection of Fig. 9 instead reveals a steady decline in line velocity. Moreover, the maximum kinetic energy determined via the radioactive heating model is in excess of that expected from the magnetar model (e.g. Usov 1992), where it is suggested that only up to $\sim 2 \times 10^{52}$ erg energy is available to power the SN (though see Metzger et al. 2015). That we find more than twice this value adds additional credence to the notion that the compact object formed during the core-collapse of the progenitor of SN 2016jca was a black hole, rather than a neutron star. This conclusion is contrary to the results of Ashall et al. (2017), who, despite finding a kinetic energy in excess $5 \times 10^{52} \mathrm{erg}$, suggest the compact object formed at the time of core-collapse was a magnetar, which is based primarily on the wide jet angle inferred from modelling their optical and X-ray LCs.

\section{The mystery of the pre-maximum $g$-band bump}

As seen in Fig. 5, the SN appears to be double peaked in the $g$-band, though intriguingly such behaviour is not observed for the other optical filters (riz). Early peaks (i.e. those occurring before the main peak) have been observed for other GRBSNe, namely SN 2006aj (Campana et al. 2006) and SN 2010bh (Cano et al. 2011b; Olivares et al. 2012). Pre-maximum bumps have also been observed for type Ic SNe not associated with a GRB, including SLSNe-Ic (Leloudas et al. 2012; Nicholl et al. 2015; Smith et al. 2016) and type Ic SN iPTF15dtg (Taddia et al. 2016). Detecting a pre-peak bump requires daily cadence, especially during the first 10 days, and a smoothly evolving AG (i.e. unlike that observed for SN 2003dh, Matheson et al. 2003). This rules out the possibility of detecting such a bump for many GRB-SNe where the observational cadence was insufficient during these times. For those GRB-SNe that were well observed, no such bump was detected (e.g. SN 1998bw, SN 2012bz, SN 2013dx, GRB 140606B). Interestingly, a slight excess of flux was found in the observer-frame $r$-band filter of SN 2013fu, associated with GRB $130215 \mathrm{~A}(z=0.479$, corresponding to restframe $\lambda=6290 /(1+z)=4253 \AA$ ), which is defined by only three data-points, and the excess was not discussed by the authors (Cano et al. 2015).

The origin of the pre-maximum flux for SN 2006aj has been debated by several authors. Campana et al. (2006) explained the achromatic pre-maximum peaks as cooling shock-heated material (from the initial shock breakout; SBO). A thermal component was also seen in the X-ray, which cooled and moved into the UV and optical regimes. In this model the observed features arose from the breakout of a shock driven by a mildly relativistic shell into a dense, and optically thick, stellar wind. An alternative model to explain the achromatic early peaks was proposed by Margutti et al. (2015) and Nakar (2015), where the breakout of the thin shell is from an extended (a few hundred solar radii) low-mass (a few hundredths of a solar mass) envelope surrounding the exploding star.

For the non-GRB type Ic SN iPTF15dtg, Taddia et al. (2016) considered several models to explain the pre-maximum peak, including a SBO cooling tail (e.g. Piro \& Nakar 2013), a magnetardriven SBO tail (Kasen et al. 2016), the extended-envelope scenario of Nakar (2015), and SN ejecta interacting with a companion star in a binary system (Kasen 2010). In the cases of type Ic SN iPTF15dtg and SLSNe-Ic LSQ14bdq (Nicholl et al. 2015) \& DES14X3TAZ (Smith et al. 2016), the extended-envelope scenario provided the most realistic explanation of the early achromatic peaks. 

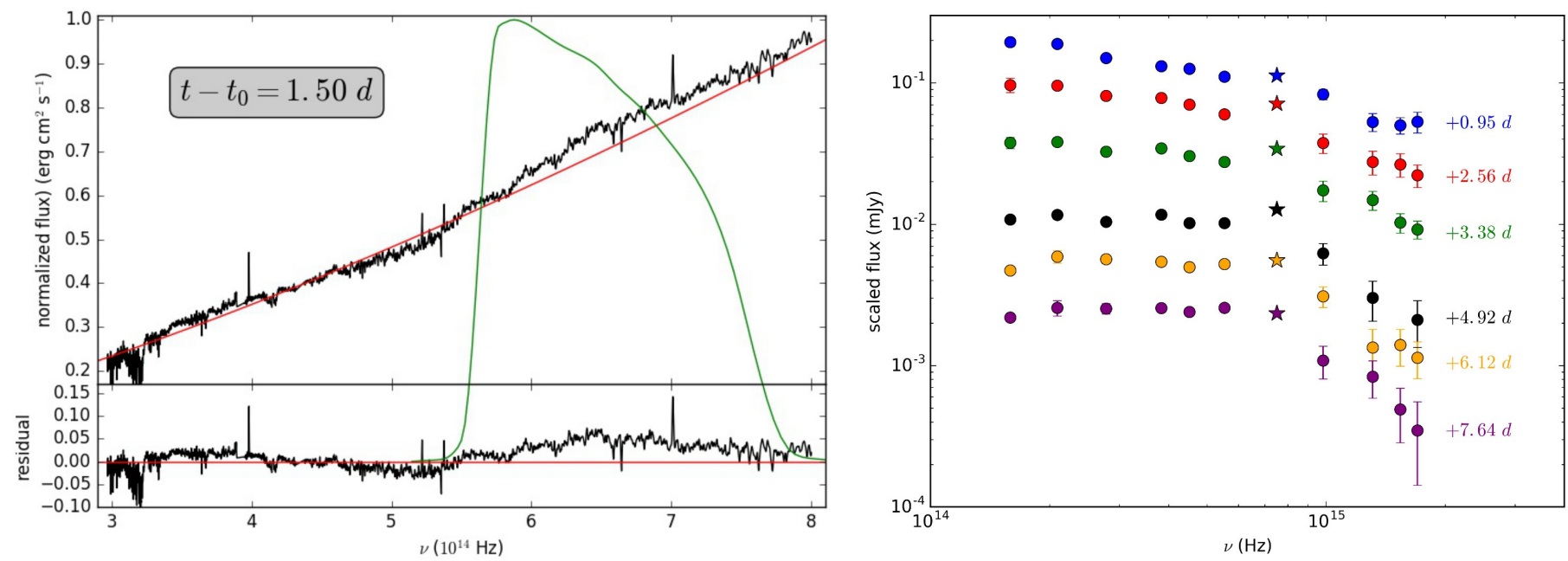

Fig. 14. Inspection of the $g$-band excess. Left: XS spectrum obtained at $t-t_{0}=1.5$ day (observer-frame). A SPL (red line) was fit to the spectrum in the wavelength range $6000 \leq \lambda \leq 9000 \AA\left(3-5 \times 10^{14} \mathrm{~Hz}\right)$. Between $3000 \sim 5500 \AA\left(5.5-8 \times 10^{14} \mathrm{~Hz}\right)$, excess above the best-fitting SPL is seen. Over-plotted is the $g$-band transmission curve, which shows that the excess occurs only in this filter. Right: optical \& UV SED evolution $(\mathrm{AG}+\mathrm{SN}+$ host), using data taken from our ground-based telescopes and Swift-UVOT. The $g$-band photometry are shown as stars. At early times $\left(t-t_{0}=0.95\right.$ day), little excess is seen in the $g$-band. However, between $t-t_{0}=2.56-6.12$ days, a clear excess is seen, which disappears by 7.64 days. Intriguingly, excess is not seen in the bluer UVOT filters.

For SN2016jca, there is one key difference with respect to the aforementioned SNe: the early peak is not achromatic, but only appears in the $g$-band. Already this rules out all of the aforementioned scenarios, all of which predict pre-maximum peaks in several filters, and not just one.

So how can this early peak be explained? The first point of interest is determining if it is even real - it could instead be a relic of an improper data-reduction and calibration method. In order to determine whether the early $g$-band peak is real, we inspected our spectra to look for evidence of any excess in the wavelength range corresponding to $g$-band, and during the same time window. The earliest spectrum presented here is the XS spectrum obtained at at $t-t_{0}=1.5$ day (observer-frame). To check for excess, we fit the entire spectrum with a SPL, (Fig. 14), where the $g$-band transmission curve is over-plotted for reference. Between $3000 \sim 5500 \AA\left(5.5-8 \times 10^{14} \mathrm{~Hz}\right)$, excess above the best-fitting SPL is clearly seen.

Thus, two different telescopes/instruments confirm that the early $g$-band excess is seen. But what about bluer wavelengths; is excess also observed? To address this, we compiled the SwiftUVOT observations obtained of SN 2016jca up to $t-t_{0}=8$ days. We then investigated several NIR to UV SEDs to check for excess at other wavelengths (right panel of Fig. 14). Six epochs are shown for $t-t_{0}=0.95,2.56,3.38,4.92,6.12$ and 7.64 days. The epoch at $t-t_{0}=0.95$ days offers little evidence for $g$ band excess, though the excess is clearly seen between $t-t_{0}=$ $2.56-6.12$ days, and then disappears by 7.64 days. Moreover, visual inspection of the SEDs reveals that only the $g$-band displays evidence of flux excess.

The cause of this $g$-band excess is not immediately obvious. It is unlikely to be related to one or more emission lines as none are observed in this wavelength region in the spectral time-series shown in Fig. 6 (though we note that Ashall et al. 2017, some excess of flux is also seen in their spectrum at $t-t_{0}=3.73$ days around $\lambda=4000 \AA$ ). Moreover, it evolves quite rapidly: it is not convincingly seen in the SED at $t-t_{0}=0.95$ day $(0.83$ days rest-frame), and has disappeared by 7.64 days (6.6 days restframe). This chromatic behaviour is not readily explained by the aforementioned theoretical models, which ultimately leaves its physical origin an unsolved mystery.

\section{The host galaxy}

The field of GRB 161219B was observed by Pan-STARRS1 in grizY prior to explosion. These images show a host galaxy that is morphologically consistent with an edge-on spiral. The GRB appears to be located close to the disk plane, at a distance of $1^{\prime \prime} .5 \pm 0$ ". 2 from the galaxy bulge, equivalent to a projected distance $3.9 \pm 0.5 \mathrm{kpc}$ at a redshift of $z=0.1475$. A recent study by Lyman et al. (2017) found the median offset of a sample of $N=39$ GRBs from their apparent host centres of $1.0 \pm 0.2 \mathrm{kpc}$, which implies that GRB 161219B occurred at a relatively further distance from its host's centre than most GRBs.

Photometry of the host was performed on these images using a circular aperture with a radius of $4{ }^{\prime \prime} 0$ that encircled the complete galaxy light visible in the Pan-STARRS1 images, and were calibrated using Pan-STARRS1 DR1 (PS1) field stars. We found AB magnitudes of: $g=21.24 \pm 0.08, r=20.62 \pm 0.07$, $i=20.63 \pm 0.08, z=20.21 \pm 0.12, Y=20.06 \pm 0.26$, which are corrected for foreground extinction. Note that these magnitudes differ from those in Table D.1, which are for a smaller aperture of 2 !' 2 .

Next, a set of galaxy templates were fit to the derived host magnitudes using LePhare (version 2.2, Arnouts et al. 1999; Ilbert et al. 2006). The templates were based on the models from Bruzual \& Charlot (2003). The photometry of the host galaxy of GRB 161219B is best fit by a galaxy template (see Fig. 15) with a stellar mass of $\log \left(M_{*} / M_{\odot}\right)=\left(8.88_{-0.10}^{+1.03}\right)$, a star-formation rate (SFR) of $0.25_{-0.17}^{+0.30} M_{\odot} \mathrm{yr}^{-1}$, an age of $\left(0.90_{-0.16}^{+5.98}\right) \times 10^{9} \mathrm{yr}$, and a negligible intrinsic extinction. We note that the constraints derived from the host galaxy photometric fit are not overly constraining due to the limited wavelength coverage.

HST imaging obtained with WFC3 (proposal \#14901, PI: A. Levan) shows the host galaxy in much more detail, which again resembles an edge-on spiral (see Fig. 1) with an elongated disk that extends $8^{\prime \prime} .5 \times 00^{\prime \prime} 8(22 \times 2 \mathrm{kpc})$, a central bulge, and 


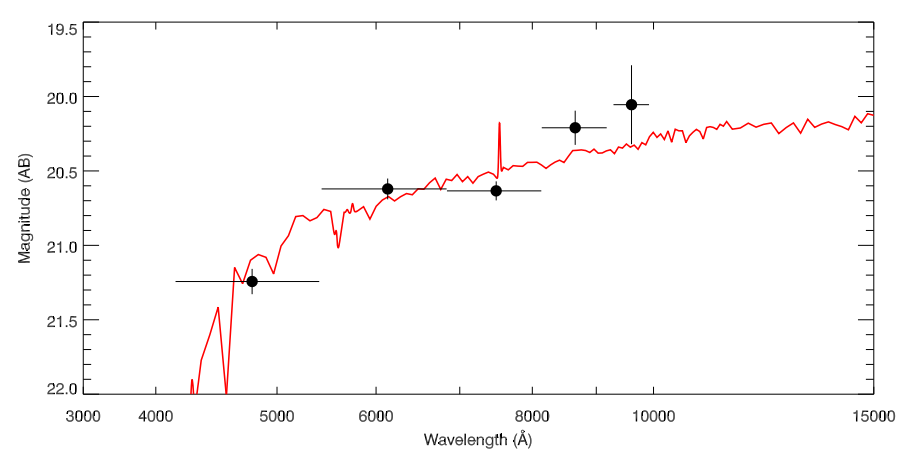

Fig. 15. Optical ( $g r i z Y$ ) photometry (for a 4!"0 circular aperture) of the host galaxy of GRB 161219B. We fit the models of Bruzual \& Charlot (2003) to the optical SED, finding best-fitting parameters of: an age of $\left(0.90_{-0.16}^{+5.98}\right) \times 10^{9} \mathrm{yr}$, a stellar mass of $\log \left(M_{*} / M_{\odot}\right)=\left(8.88_{-0.10}^{+1.03}\right)$, a SFR of $0.25_{-0.17}^{+0.30} M_{\odot} \mathrm{yr}^{-1}$ and negligible intrinsic extinction.

at least two distinctive knots on the disk, possibly due to starforming (SF) regions. In the HST image we measure a distance of SN 2016jca of $11^{\prime \prime 30} \pm 00^{\prime \prime} .05(3.38 \pm 0.13 \mathrm{kpc})$ to the centre of the galaxy (NB: defining the precise location of galactic core poses the largest source of uncertainty in this calculation).

The spectra of SN 2016jca obtained on the 22-January-2017 has the slit positioned along the edge-on galaxy (see Table C.1). We extracted the 1D spectra in fixed-width bins (1'.5) in steps of 0 '.75 and analysed them separately. The flux values and physical properties in the different regions are listed in Table 3, while the properties along the slit are plotted in Fig. 16. The metallicity was calculated using the N2 parameter (Marino et al. 2013), while the specific SFR (sSFR) weighted by the (rest-frame) $B$ band magnitude (see Table 3 ). The latter values were determined from the host photometry (in rest frame $B$-band) resolved for each of the host galaxy bins. Given the higher spatial resolution of the HST images with respect to the ground based ones, the former were deemed more appropriate to be used to estimate the resolved photometry. A magnitude equivalent to the restframe Johnson $B$-band was estimated using the flux calibrated spectrum of the host galaxy. This value was then used to scale the flux of the galaxy in the HST image (zero order of the slit-less L200LP grism observation, whose central wavelength is around $5000 \AA$ ).

There is little variation across the galaxy, which is not surprising given its edge-on viewing angle, hence the spectra are all dominated by the light from the outer spiral arms. Neither the SFR nor the metallicity are extreme/peculiar at the SN position compared to the rest of the host (see Table 3). Indeed, the SFR and SSFR are highest in the SF region at the opposite side of the galaxy. The integrated host spectrum has a metallicity of 0.4 solar $(12+\log (\mathrm{O} / \mathrm{H})=8.28)$ and a SFR of $0.17 M_{\odot} \mathrm{yr}^{-1}$, consistent with the value obtained from the SED fitting of the host. The sSFR of the entire galaxy is $2.91 M_{\odot} \mathrm{yr}^{-1}\left(L / L_{*}\right)^{-1}$, and we find a mass-weighted SFR of $0.18 \mathrm{Gyr}^{-1}$.

We find that the mass and the sSFR are consistent with the mean value of GRB hosts at $z<0.5$ (e.g. Perley et al. 2016; Schulze et al. 2016), but the SFR is smaller than the average. Krühler et al. (2015) find that the SFR increases with redshift, which is partially an effect of increasing host mass with redshift since SFR and stellar mass are known to be correlated (with exceptions), i.e. the so-called SFR-main sequence (e.g. Elbaz et al. 2007, Bouwens et al. 2012, for low and high redshifts respectively). The stellar mass of GRB 161219B's host is somewhat above-average for hosts at a similar redshift (see e.g.

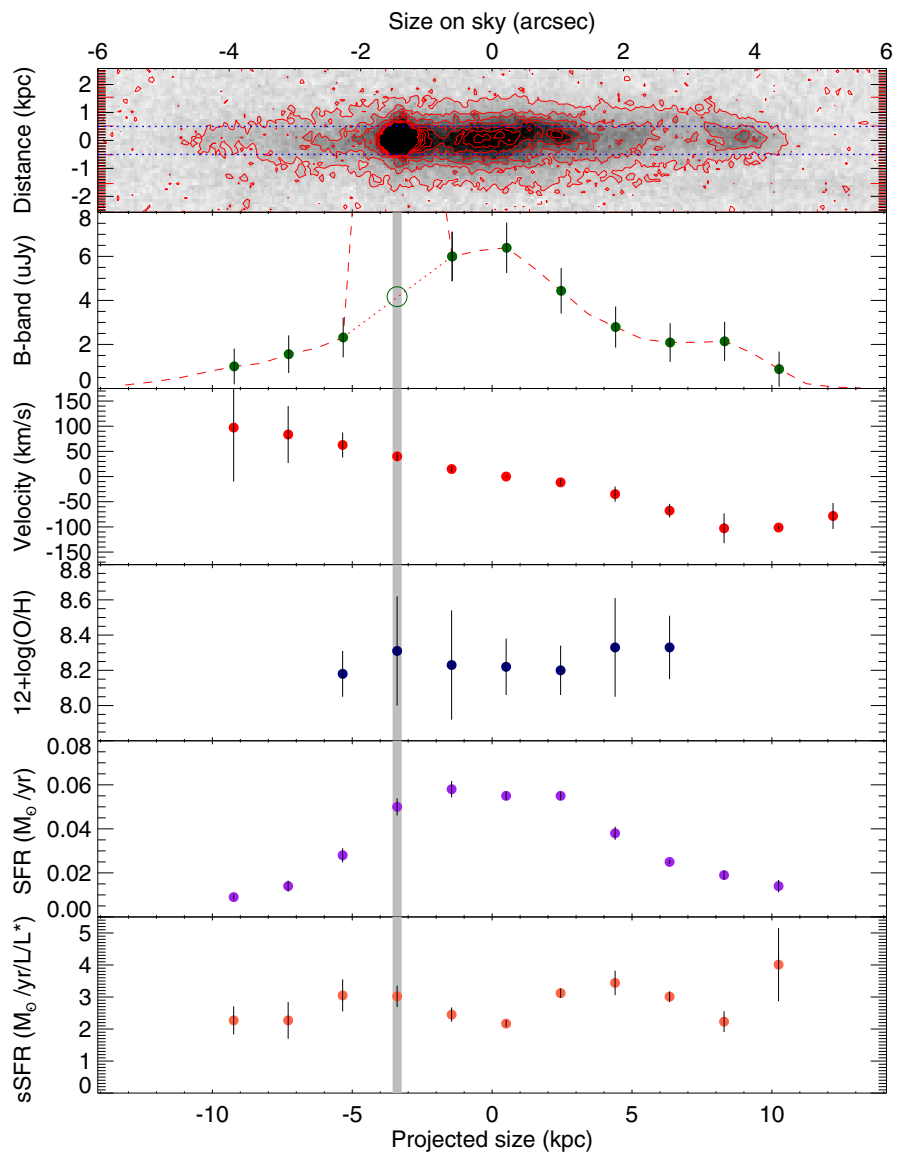

Fig. 16. Host galaxy properties along the slit on the GTC observation of the 26 January 2017, as compared to the HST image. The first panel shows one of the HST images with contours superposed in red and the position and width of the GTC slit indicated by the dotted blue lines. The second panel is the $B$-band flux profile along the slit using the flux obtained from the 0th order L200LP grism from HST as reference. The third panel shows the relative velocity measured using the [O II], [O III] and $\mathrm{H} \alpha$ lines. The fourth panel shows the metallicity using the N2 parameter (Marino et al. 2013). The fifth panel shows the SFR derived from the $\mathrm{H} \alpha$ emission. The last panel is the SFR weighted by the $B$-band magnitude in panel 2 . The vertical grey line marks the location of SN 2016jca.

Krühler et al. 2015; Perley et al. 2016; Vergani et al. 2015), so one might expect a higher SFR that measured here; instead we find the opposite, i.e. that the galaxy has one of the lowest SFRs measured for any GRB host (see Krühler et al. 2015). Edge-on galaxies often show lower measured SFRs as part of the light is hidden behind dust lanes, however, this does not seem to be an issue here as we measure very low extinction in the SED fit.

The metallicity of the host is rather typical for a longduration GRB host (Krühler et al. 2015), which appears to support the notion of previous results that GRB hosts do not show an extremely low metallicity preference, as required by most current GRB progenitor models. In Fig. 17 we plot the line ratios from the different parts of the galaxy into the Baldwin-PhillipsTerlevich (BPT) diagram, which allows us to distinguish between SF- and AGN-driven regions, and to some extent the age and metallicity of each region, depending on the ionisation parameter. In general, younger and more metal-poor galaxies are found towards the upper left of the BPT diagram. All regions within the host of GRB 161219B occupy very similar regions in the diagram, and they are well within the part of the diagram 
Table 3. GRB 161219B host galaxy emission lines and properties.

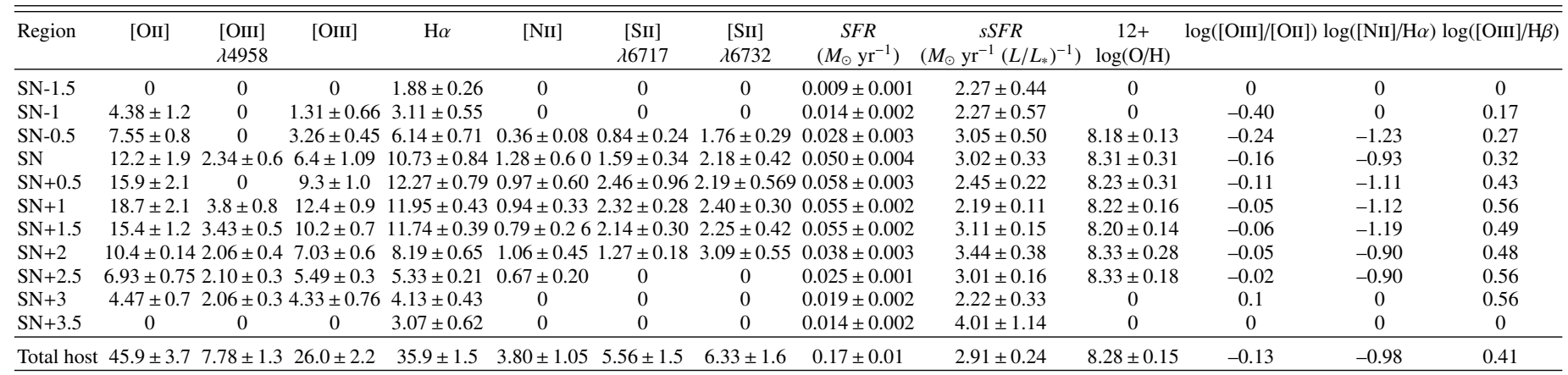

Notes. The regions mark the centre of the extracted spectrum relative to the SN location in units of steps (one step corresponds to 1 1"5), hence the spectra are overlapping. Fluxes are in units of $10^{-17} \mathrm{erg} \mathrm{cm}^{-2} \mathrm{~s}^{-1}$. For the ratio [OIII]/H $\beta$, the flux of $\mathrm{H} \beta$ is derived from $\mathrm{H} \alpha$ assuming zero extinction and Case B recombination $(\mathrm{H} \alpha / \mathrm{H} \beta=2.76)$. Errors in the SFRs only reflect the error in the $\mathrm{H} \alpha$ flux, while the sSFR also considers the error in the $B$-band magnitude.

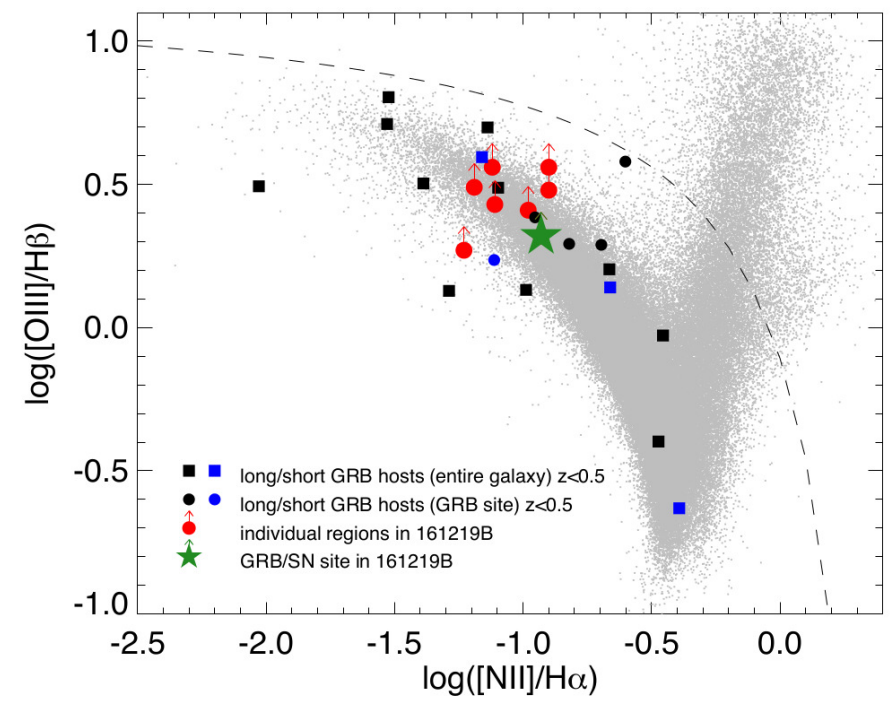

Fig. 17. BPT diagram showing the different regions in the host of GRB 161219B, where the GRB site is highlighted by the green star. Values presented here are only lower limits as $\mathrm{H} \beta$ could not be measured due to its location on top of an atmospheric emission line. Grey dots are galaxies from SDSS (DR9), while the squares and dots are other GRB hosts/GRB sites (the latter for cases of resolved galaxies) at $z<0.5$. The dashed line marks the dividing line between SF regions and those dominated by AGN activity. Data are taken from Christensen et al. (2008), Berger (2009), Han et al (2010), Levesque et al. (2010), Perley et al. (2012), Thöne et al. (2014), de Ugarte Postigo et al. (2014b), Schulze et al. (2014), Stanway et al. (2015), Krühler et al. (2015).

typically occupied by GRB hosts at low redshifts, but somewhat more extreme than the bulk of SF galaxies found in the SDSS.

In summary, the host is a rather typical GRB host at its redshift, with the only difference of a relatively lower SFR and sSFR. Most GRB hosts at low $z$ seem to be dwarf galaxies, but there is a growing fraction of GRBs occurring in spiral galaxies (e.g. GRB 980425 Fynbo et al. 2000; Christensen et al. 2008; Krühler et al. 2017; GRB 060505 Thöne et al. 20145 ; GRB 111005A Michałowski et al. 2016), though all of them have small stellar masses. Interestingly, in two of those spiral

\footnotetext{
5 Although there is debate regarding the long/short nature of this GRB, e.g. Ofek et al. (2007).
}

hosts (GRBs 060505 and 111005A), no SNe associated with the GRBs were detected despite their very low redshift (Fynbo et al. 2006; Michałowski et al. 2016). However, they both were rather different galaxies: GRB 060505 has a low metallicity and high SFR, particularly at the GRB site (Thöne et al. 2008, 2014), while the host of GRB 111005A has a super-solar metallicity and an even lower sSFR than GRB 161219B (Michałowski et al. 2016).

\section{Discussion and conclusions}

We presented optical and NIR photometry, and optical spectroscopy of GRB 161219B and its associated SN 2016jca. The early optical/NIR AG is characterised by a shallow decline, with a temporal index of $\alpha=0.6-0.8$. The shallower index results from the NIR LCs, which are contaminated with host flux. Instead, the host-subtracted optical data in griz have a steeper decay index of $\alpha \approx 0.8$, which is precisely that found from modelling the X-ray LC. We also modelled several early NIR to $\mathrm{X}$-ray SEDs with a BPL, finding spectral indices of $\beta_{\text {opt }} \approx 0.45$ and $\beta_{\mathrm{X}} \approx 0.95$, with a break frequency of $\nu_{\mathrm{BB}} \approx 1.75 \times 10^{15} \mathrm{~Hz}$, i.e. in the mid-to-far UV range.

The optical and X-ray spectral indices are consistent with synchrotron emission arising from a fireball colliding with circumburst material (e.g. Sari et al. 1998). In this scenario, electrons accelerated by the fireball are cooling slowly, with an electron index of $p=1$.9. In our modelling, we find that the cooling break lies between the optical and the X-rays, which is the same conclusion found by Ashall et al. (2017). The values of the spectral and temporal indices are consistent with a fireball colliding with a homogeneous medium: from the closure relations between $\alpha$ and $\beta$ (e.g. Sari et al. 1999; Chevalier \& Li 2000), our measured values of $\beta$ suggest a temporal index in the optical of 0.68 (as compared to the measured values of $0.61-0.86$ ) and in the X-rays of 0.93 (as compared to the measured value of 0.79 ) before the jet break, and of 1.90 after the jet break (compared to the measured value of 1.93). We see that, although there are mild inconsistencies in the temporal slopes (which could be due to a departure from a uniform medium, i.e. it is slightly clumpy) the consistency between model and data is very good. We also note that the data are inconsistent with a stellar wind density profile surrounding the GRB progenitor, as it would require temporal decays in the optical of 1.18 , which are inconsistent with the measured value. 
When fitting the NIR to X-ray SEDs, a strong thermal component was found at $t-t_{0}=0.26$ days $\left(T_{\mathrm{BB}} \approx 0.16 \times\right.$ $10^{6} \mathrm{~K}$ ), which contributed roughly $70 \%$ of the total observed flux (Sect. 5). The strength of the BB component decreased to $<10 \%$ at +1.47 days, and disappeared completely by +2.55 days The radius of the thermal component was found to be $R_{\mathrm{BB}} \approx$ $6 \times 10^{14} \mathrm{~cm}$, which is much larger than those found for e.g. GRB 060218 and GRB 120422A by almost one order of magnitude. Interestingly though, the NIR to X-ray SED of GRB $120422 \mathrm{~A}$ at $t-t_{0}=0.267$ days had a similar temperature to that found for GRB 161219B at a similar post-explosion epoch $\left(T_{\mathrm{BB}} \approx 0.19 \times 10^{6} \mathrm{~K}\right)$, which was interpreted by Schulze et al. (2014) as emission arising from a cooling, expanding stellar envelope after the passage of the shock breakout through it. No premaximum bumps were observed for SN 2012bz, meaning a similar interpretation of the thermal component for GRB 161219B is appealing.

Next, we demonstrated that SN 2016jca was less luminous and evolved more rapidly than the comparison/template SN 1998bw. Using the blueshifted line velocities of Fe II $\lambda 5169$ as a proxy for the photospheric velocity, we find that SN 2016jca has a peak photospheric velocity of $v_{\mathrm{ph}}=297000 \pm 1500 \mathrm{~km} \mathrm{~s}^{-1}$. This is more than $1 \sigma$ times more rapid than the "typical" GRB$\mathrm{SN}$ (Cano et al. 2017), which has $v_{\mathrm{ph}}=20000 \mathrm{~km} \mathrm{~s}^{-1}$, with a standard deviation of $\sigma=8000 \mathrm{~km} \mathrm{~s}^{-1}$. Such a rapid expansion velocity was also confirmed by Ashall et al. (2017).

To determine what powers the luminosity of SN 2016jca, we considered two models: a radioactive heating model and a magnetar model. The latter model was individually fit to both the $\mathrm{X}$-ray and the $r$-band luminosity LCs, and the best-fitting parameters for the spin period and magnetic field determined from both frequency regimes were inconsistent with each other. Moreover, the model was unable to reproduce the shallow decay in both the X-ray and optical LCs seen at late times. The radioactiveheating model provided a much better fit to the griz bolometric LC, where we found a nickel mass of $M_{\mathrm{Ni}}=0.22 \pm 0.08 M_{\odot}$, and ejecta mass of $M_{\mathrm{ej}}=5.8 \pm 0.3 M_{\odot}$, and a kinetic energy of $E_{\mathrm{K}}=5.1 \pm 0.8 \times 10^{52} \mathrm{erg}$. We also found a $\gamma$-ray opacity of $\kappa_{\gamma}=0.034 \mathrm{~cm}^{2} \mathrm{~g}^{-1}$, which falls within the range of $\gamma$-ray opacities found by Wheeler et al. (2015) for a sample of $N=20 \mathrm{SNe}$ Ibc.

The kinetic energy found here is well in excess of that expected for an explosion powered by a magnetar, where a maximum value of $E_{\mathrm{K}} \leq 2 \times 10^{52} \mathrm{erg}$ has been suggested (Usov 1992; Mazzali et al. 2014). The SN's energetics were also confirmed by Ashall et al. (2017) to be more than $5 \times 10^{52} \mathrm{erg}$. The results of our magnetar model, as well as the Fe II $\lambda 5169$ velocity evolution (which is not flat, as predicted by 1D magnetar models) also argue against a magnetar powering any phases of GRB 161219B/SN 2016jca. Instead, the energetics found here are more indicative of a black hole being formed at the time of core-collapse. This conclusion is at odds to that of Ashall et al. (2017), who despite this energetic constraint, argue that a magnetar may likely be powering the $\mathrm{SN}$ outflow, which is based at least on part on the large jet angle inferred from their observations.

When analysing the $\gamma$-ray properties of GRB 161219B, we found that its isotropic-equivalent $\gamma$-ray energy is $E_{\gamma \text {,iso }} \approx 8.5 \times$ $10^{49} \mathrm{erg}$, which when we consider its duration $\left(T_{90}=6.9 \mathrm{~s}\right), \mathrm{im}-$ plies that it is an intermediate-luminosity GRB. Moreover, along with its peak energy $\left(E_{\mathrm{p}} \approx 106 \mathrm{keV}\right)$, GRB $161219 \mathrm{~B}$ is an outlier in the Amati relation. We found the same conclusion when we considered the rest-frame energetics as constrained from both Swift-BAT and Konus-Wind.

The host galaxy of GRB 161219B/SN 2016jca appears to be an edge-on spiral, whose photometric (griz $Y$ ) SED is consistent with a galaxy of age $\approx 1 \mathrm{Gyr}$, a mass of $\approx 7.6 \times 10^{8} M_{\odot}$, a SFR of $\approx 0.25 M_{\odot} \mathrm{yr}^{-1}$, and negligible intrinsic extinction. Inspection of HST images reveals that the GRB occurred at a projected distance of $\approx 3.4 \mathrm{kpc}$ from the host's center. We divided the integrated host spectrum into discrete bins, and determined the metallicity, SFR and sSFR as a function of position along the galactic disk. There is little variation in these values across the galaxy. Neither the metallicity nor the SFR is extreme at the GRB's position compared with the rest of the host. The SFR and SSFR are largest in the SF region at the opposite side of the galaxy. From the integrated host spectrum we find a metallicity of $\approx 0.4$ solar, a modest SFR of $\approx 0.17 M_{\odot} \mathrm{yr}^{-1}$ and a sSFR of $\approx 2.91 M_{\odot} \mathrm{yr}^{-1} L L_{*}^{-1}$. Both the mass and sSFR are commensurate with GRB hosts at $z<0.5$. The derived host-integrated metallicity is perfectly commensurate with those of other GRB hosts.

Finally, we report on the presence of a chromatic, premaximum bump in the observer-frame $g$-band filter. At +1.5 days, an excess of flux in the $g$-band is seen in the XS spectrum. The evolution of the $g$-band excess was shown by a timesequence of UV to NIR SEDs, which appears to peak around 3-5 days (observer-frame), and disappears by +7.6 days. While pre-maximum bumps have been seen for GRB-SNe, SLSNe-Ic and non-GRB SNe Ic, their achromatic behaviour means that the analytical models used to describe their physics (which are usually best-fit by the low-mass extended-envelope model of Nakar 2015) do not apply in this case. After demonstrating that the $g$-band excess is real, we are unable to conclude on its physical origin.

In conclusion, SN 2016jca is only the seventh GRB-SNe to have been detected within $1 \mathrm{Gpc}$, and has therefore provided a rare but excellent chance to determine both its physical and observations properties. In relation to the general GRB-SN population, its nickel mass and ejecta mass are perfectly commensurate. However, its photospheric velocity appears to be more rapid than most GRB-SNe, which in turn implies a large kinetic energy. Its large kinetic energy, taken in tandem with the results of the magnetar modelling and the velocity evolution, argue against a magnetar powering this event, and instead it is more likely that a black hole was formed at the time of core-collapse.

Acknowledgements. L.I., C.T., Z.C., A.d.U.P. and D.A.K. acknowledge support from the Spanish research project AYA 2014-58381-P. C.T. and A.d.U.P. furthermore acknowledge support from Ramón y Cajal fellowships RyC-201209984 and RyC-2012-09975. D.A.K. and Z.C. acknowledge support from Juan de la Cierva Incorporación fellowships IJCI-2015-26153 and IJCI-2014-21669. R.S.R. is supported by a 2016 BBVA Foundation Grant for Researchers and Cultural Creators. T.-W. Chen and T. Krühler acknowledge the support through the Sofia Kovalevskaja Award to P. Schady from the Alexander von Humboldt Foundation of Germany. D.M. acknowledges support from the Instrument center for Danish Astrophysics (IDA). Our analysis was based on: (1) Observations collected at the European Organisation for Astronomical Research in the Southern Hemisphere under ESO programme 098.A-0055(A). (2) Observations made with the Nordic Optical Telescope, operated by the Nordic Optical Telescope Scientific Association at the Observatorio del Roque de los Muchachos, La Palma, Spain, of the Instituto de Astrofisica de Canarias. (3) Observations collected at the European Organisation for Astronomical Research in the Southern Hemisphere, Chile as part of PESSTO, (the Public ESO Spectroscopic Survey for Transient Objects Survey) ESO program 188.D-3003, 191.D-0935, 197.D-1075. (4) Development of CIRCE was supported by the University of Florida and the National Science Foundation (grant AST-0352664), in collaboration with IUCAA. (5) Part of the funding for GROND (both hardware as well as personnel) was generously granted from the Leibniz-Prize to Prof. G. Hasinger (DFG grant HA 1850/28-1). (6) The Pan-STARRS1 Surveys (PS1) and the PS1 public 
science archive have been made possible through contributions by the Institute for Astronomy, the University of Hawaii, the Pan-STARRS Project Office, the Max-Planck Society and its participating institutes, the Max Planck Institute for Astronomy, Heidelberg and the Max Planck Institute for Extraterrestrial Physics, Garching, The Johns Hopkins University, Durham University, the University of Edinburgh, the Queen's University Belfast, the Harvard-Smithsonian Center for Astrophysics, the Las Cumbres Observatory Global Telescope Network Incorporated, the National Central University of Taiwan, the Space Telescope Science Institute, the National Aeronautics and Space Administration under Grant No. NNX08AR22G issued through the Planetary Science Division of the NASA Science Mission Directorate, the National Science Foundation Grant No. AST1238877, the University of Maryland, Eotvos Lorand University (ELTE), the Los Alamos National Laboratory, and the Gordon and Betty Moore Foundation. (7) Observations made with the NASA/ESA Hubble Space Telescope, obtained at the Space Telescope Science Institute, which is operated by the Association of Universities for Research in Astronomy, Inc., under NASA contract NAS 526555. These observations are associated with program 14901.

\section{References}

Alard, C. 2000, A\&AS, 144, 363

Alard, C., \& Lupton, R. H. 1998, ApJ, 503, 325

Alexander, K. D., Laskar, T., \& Berger, E. 2016, GRB Coordinates Network, 20313, 1

Amati, L., Frontera, F., Tavani, M., et al. 2002, A\&A, 390, 81

Arnaud, K. A. 1996, Astronomical Data Analysis Software and Systems V, ASP Conf. Ser., 101, 17

Arnett, W. D. 1982, ApJ, 253, 785

Arnouts, S., Cristiani, S., Moscardini, L., et al. 1999, MNRAS, 310, 540

Ashall, C., Pian, E., Mazzali, P. A., et al. 2017, ArXiv e-prints [arXiv: 1702.04339]

Barkov, M. V., \& Komissarov, S. S. 2011, MNRAS, 415, 944

Bazin, G., Ruhlmann-Kleider, V., Palanque-Delabrouille, N., et al. 2011, A\&A, 534, A43

Beardmore, A. P., Evans, P. A., Goad, M. R., \& Osborne, J. P. 2016, GRB Coordinates Network, 20297, 1

Berger, E. 2009, ApJ, 690, 231

Bersten, M. C., Benvenuto, O. G., Orellana, M., \& Nomoto, K. 2016, ApJ, 817, L8

Beuermann, K., Hessman, F. V., Reinsch, K., et al. 1999, A\&A, 352, L26

Bouwens, R. J., Illingworth, G. D., Oesch, P. A., et al. 2012, ApJ, 754, 83

Bromberg, O., Nakar, E., \& Piran, T. 2011, ApJ, 739, L55

Bruzual, G., \& Charlot, S. 2003, MNRAS, 344, 1000

Bucciantini, N., Quataert, E., Arons, J., Metzger, B. D., \& Thompson, T. A. 2007, MNRAS, 380, 1541

Buckley, D. A. H., Hamanowicz, A., Martin-Carrillo, A., et al. 2016, GRB Coordinates Network, 20322, 1

Bufano, F., Pian, E., Sollerman, J., et al. 2012, ApJ, 753, 67

Campana, S., Mangano, V., Blustin, A. J., et al. 2006, Nature, 442, 1008

Cano, Z. 2013, MNRAS, 434, 1098

Cano, Z. 2014, ApJ, 794, 121

Cano, Z., Bersier, D., Guidorzi, C., et al. 2011a, MNRAS, 413, 669

Cano, Z., Bersier, D., Guidorzi, C., et al. 2011b, ApJ, 740, 41

Cano, Z., Maeda, K., \& Schulze, S. 2014a, MNRAS, 438, 2924

Cano, Z., de Ugarte Postigo, A., Pozanenko, A., et al. 2014b, A\&A, 568, 19

Cano, Z., Jakobsson, P., \& Pall Geirsson, O. 2014c, ArXiv e-prints [arXiv: 1409.3570]

Cano, Z., de Ugarte Postigo, A., Perley, D., et al. 2015, MNRAS, 452, 1535

Cano, Z., Johansson Andreas, K. G., \& Maeda, K. 2016, MNRAS, 457, 2761

Cano, Z., Wang, S.-Q., Dai, Z.-G., \& Wu, X.-F. 2017, Adv., Astron., 2017, 8929054

Cappellaro, E., Mazzali, P. A., Benetti, S., et al. 1997, A\&A, 328, 203

Cardelli, J. A., Clayton, G. C., \& Mathis, J. S. 1989, ApJ, 345, 245

Chambers, K. C., Magnier, E. A., Metcalfe, N., et al. 2016, ArXiv e-prints [arXiv: 1612.05560]

Chatzopoulos, E., Wheeler, J. C., Vinko, J., et al. 2011, ApJ, 729, 143

Chen, T.-W., Smartt, S. J., Jerkstrand, A., et al. 2015, MNRAS, 452, 1567

Chen, T.-W., Greiner, J., Klose, S., et al. 2017, GRB Coordinates Network, 20380, 1

Chevalier, R. A. 1992, ApJ, 394, 599

Chevalier, R. A., \& Li, Z.-Y. 2000, ApJ, 536, 195

Christensen, L., Vreeswijk, P. M., Sollerman, J., et al. 2008, A\&A, 490, 45

Chugai, N. N. 2000, Astron. Lett., 26, 797

Cummings, J. R., Barthelmy, S. D., Baumgartner, W. H., et al. 2012, GRB Coordinates Network, 13481, 1
D’Ai, A., Kennea, J. A., Krimm, H. A., et al. 2016, GRB Coordinates Network, 20296, 1

D’Elia, V., Pian, E., Melandri, A., et al. 2015, A\&A, 577, A116

Dall'Osso, S., Stratta, G., Guetta, D., et al. 2011, A\&A, 526, A121

Deng, J., Tominaga, N., Mazzali, P. A., Maeda, K., \& Nomoto, K. 2005, ApJ, 624,898

Dessart, L., \& Hillier, D. J. 2005, A\&A, 439, 671

Dessart, L., Hillier, D. J., Woosley, S., et al. 2015, MNRAS, 453, 2189

Elbaz, D., Daddi, E., Le Borgne, D., et al. 2007, A\&A, 468, 33

Finkbeiner, D. P., Schlafly, E. F., Schlegel, D. J., et al. 2016, ApJ, 822, 66

Flewelling, H. A., Magnier, E. A., Chambers, K. C., et al. 2016, ArXiv e-prints [arXiv: 1612.05243]

Fong, W., \& Milne, P. 2016, GRB Coordinates Network, 20332, 1

Frederiks, D., Golenetskii, S., Aptekar, R., et al. 2016, GRB Coordinates Network, 20323, 1

Friis, M., \& Watson, D. 2013, ApJ, 771, 15

Fujiwara, T., Saito, Y., Tachibana, Y., et al. 2016, GRB Coordinates Network, 20314, 1

Fukugita, M., Shimasaku, K., \& Ichikawa, T. 1995, PASP, 107, 945

Fynbo, J. U., Holland, S., Andersen, M. I., et al. 2000, ApJ, 542, L89

Fynbo, J. P. U., Watson, D., Thöne, C. C., et al. 2006, Nature, 444, 1047

Fynbo, J. P. U., Jakobsson, P., Prochaska, J. X., et al. 2009, ApJS, 185, 526

Gal-Yam, A. 2012, Science, 337, 927

Galama, T. J., Vreeswijk, P. M., van Paradijs, J., et al. 1998a, Nature, 395, 670

Garner, A., Stelter, R. D., Eikenberry, S. S., et al. 2014, Proc. SPIE, 9147, 91474A

Gehrels, N., Chincarini, G., Giommi, P., et al. 2004, ApJ, 611, 1005

Golenetskii, S., Aptekar, R., Frederiks, D., et al. 2015, GRB Coordinates Network, 18198, 1

Gompertz, B. P., \& Fruchter, A. S. 2017, ApJ, 839, 49

Greiner, J., Bornemann, W., Clemens, C., et al. 2008, PASP, 120, 405

Greiner, J., Mazzali, P. A., Kann, D. A., et al. 2015, Nature, 523, 189

Guidorzi, C., Kobayashi, S., Steele, I. A., Gomboc, A., \& Mundell, C. G. 2016, GRB Coordinates Network, 20300, 1

Han, X. H., Hammer, F., Liang, Y. C., et al. 2010, A\&A, 514, A24

Hjorth, J. 2013, Phil. Trans. R. Soc. London Ser. A, 371, 20120275

Hjorth, J., \& Bloom, J. S. 2012, in Gamma-Ray Bursts, Cambridge Astrophysics Series 51, eds. C. Kouveliotou, R. A. M. J. Wijers, \& S. Woosley (Cambridge: Cambridge University Press), 169

Hjorth, J., Sollerman, J., Møller, P., et al. 2003, Nature, 423, 847

Ilbert, O., Arnouts, S., McCracken, H. J., et al. 2006, A\&A, 457, 841

Inserra, C., Smartt, S. J., Jerkstrand, A., et al. 2013, ApJ, 770, 128

Kann, D. A., Schady, P., Olivares E., F., et al. 2016, ArXiv e-prints [arXiv: 1606.06791]

Kasen, D. 2010, ApJ, 708, 1025

Kasen, D., \& Bildsten, L. 2010, ApJ, 717, 245

Kasen, D., Metzger, B. D., \& Bildsten, L. 2016, ApJ, 821, 36

Kleinmann, S. G., Lysaght, M. G., Pughe, W. L., et al. 1994, Ap\&SS, 217, 11

Klose, S., Greiner, J., Rau, A., et al. 2004, AJ, 128, 1942

Klose, S., Greiner, J., Fynbo, J., et al. 2012, Central Bureau Electronic Telegrams, 3200, 1

Krühler, T., Küpcü Yoldaş, A., Greiner, J., et al. 2008, ApJ, 685, 376

Krühler, T., Malesani, D., Fynbo, J. P. U., et al. 2015, A\&A, 581, A125

Krühler, T., Wiseman, P., \& Greiner, J. 2016, GRB Coordinates Network, 20299, 1

Krühler, T., Kuncarayakti, H., Schady, P., et al. 2017, A\&A, 602, A85

Kumar, P., \& Panaitescu, A. 2000, ApJ, 541, L51

Laskar, T., Alexander, K. D., \& Berger, E. 2016, GRB Coordinates Network, 20328, 1

Lasky, P. D., Leris, C., Rowlinson, A., \& Glampedakis, K. 2017, ApJ, 843, L1

Leloudas, G., Chatzopoulos, E., Dilday, B., et al. 2012, A\&A, 541, A129

Levan, A. J., Tanvir, N. R., Starling, R. L. C., et al. 2014, ApJ, 781, 13

Levesque, E. M., Berger, E., Kewley, L. J., \& Bagley, M. M. 2010, AJ, 139, 694

Li, X., \& Hjorth, J. 2014, ArXiv e-prints [arXiv: 1407. 3506]

Li, X., Hjorth, J., \& Wojtak, R. 2014, ApJ, 796, L4

Lyman, J. D., Bersier, D., \& James, P. A. 2014, MNRAS, 437, 3848

Lyman, J. D., Levan, A. J., Tanvir, N. R., et al. 2017, MNRAS,

MacFadyen, A. I., \& Woosley, S. E. 1999, ApJ, 524, 262

Maeda, K., Nakamura, T., Nomoto, K., et al. 2002, ApJ, 565, 405

Maeda, K., Mazzali, P. A., \& Nomoto, K. 2006, ApJ, 645, 1331

Malesani, D., Tagliaferri, G., Chincarini, G., et al. 2004, ApJ, 609, L5

Margutti, R., Guidorzi, C., Lazzati, D., et al. 2015, ApJ, 805, 159

Marino, R. A., Rosales-Ortega, F. F., Sánchez, S. F., et al. 2013, A\&A, 559, A114

Marshall, F. E., \& D’Ai, A. 2016, GRB Coordinates Network, 20306, 1

Martin, M. J. 1987, Nuclear Data Sheets, 58, 67

Martin-Carrillo, A., Murphy, D., Hanlon, L., et al. 2016, GRB Coordinates Network, 20305, 1 
Martone, R., Izzo, L., \& Della Valle, M. 2017, A\&A, submitted Matheson, T., Garnavich, P. M., Stanek, K. Z., et al. 2003, ApJ, 599, 394

Mazaeva, E., Mokhnatkin, A., Pozanenko, A., Volnova, A., \& Molotov, I. 2016, GRB Coordinates Network, 20309, 1

Mazzali, P. A., Nomoto, K., Patat, F., \& Maeda, K. 2001, ApJ, 559, 1047

Mazzali, P. A., Deng, J., Nomoto, K., et al. 2006, Nature, 442, 1018

Mazzali, P. A., McFadyen, A. I., Woosley, S. E., Pian, E., \& Tanaka, M. 2014, MNRAS, 443, 67

Metzger, B. D., Margalit, B., Kasen, D., \& Quataert, E. 2015, MNRAS, 454, 3311

Michałowski, M. J., Xu, D., Stevens, J., et al. 2016, ArXiv e-prints [arXiv: 1610.06928]

Modjaz, M., Li, W., Butler, N., et al. 2009, ApJ, 702, 226

Modjaz, M., Liu, Y. Q., Bianco, F. B., \& Graur, O. 2016, ApJ, 832, 108

Nakar, E. 2015, ApJ, 807, 172

Nayana, A. J., \& Chandra, P. 2016, GRB Coordinates Network, 20344, 1

Nicholl, M., Smartt, S. J., Jerkstrand, A., et al. 2013, Nature, 502, 346

Nicholl, M., Smartt, S. J., Jerkstrand, A., et al. 2015, ApJ, 807, L18

Ofek, E. O., Cenko, S. B., Gal-Yam, A., et al. 2007, ApJ, 662, 1129

Olivares E., F., Greiner, J., Schady, P., et al. 2012, A\&A, 539, A76

Ostriker, J. P., \& Gunn, J. E. 1971, ApJ, 164, L95

Page, K. L., Starling, R. L. C., Fitzpatrick, G., et al. 2011, MNRAS, 416, 2078

Palmer, D. M., Barthelmy, S. D., Cummings, J. R., et al. 2015, GRB Coordinates Network, 18157, 1

Palmer, D. M., Barthelmy, S. D., Cummings, J. R., et al. 2016, GRB Coordinates Network, 20308, 1

Patat, F., Cappellaro, E., Danziger, J., et al. 2001, ApJ, 555, 900

Pei, Y. C. 1992, ApJ, 395, 130

Perley, D. A., Modjaz, M., Morgan, A. N., et al. 2012, ApJ, 758, 122

Perley, D. A., Krühler, T., Schulze, S., et al. 2016, ApJ, 817, 7

Phillips, M. M. 1993, ApJ, 413, L105

Pian, E., Mazzali, P. A., Masetti, N., et al. 2006, Nature, 442, 1011

Piran, T. 2004, Rev. Mod. Phys., 76, 1143

Piro, A. L., \& Nakar, E. 2013, ApJ, 769, 67

Piro, L., Troja, E., Gendre, B., et al. 2014, ApJ, 790, L15

Price, P. A., Berger, E., Kulkarni, S. R., et al. 2002, ApJ, 573, 85

Quimby, R. M., Kulkarni, S. R., Kasliwal, M. M., et al. 2011, Nature, 474, 487

Rowlinson, A., O’Brien, P. T., Metzger, B. D., Tanvir, N. R., \& Levan, A. J. 2013 , MNRAS, 430, 1061

Sari, R., Piran, T., \& Narayan, R. 1998, ApJ, 497, L17

Sari, R., Piran, T., \& Halpern, J. P. 1999, ApJ, 519, L17

Schady, P., Page, M. J., Oates, S. R., et al. 2010, MNRAS, 401, 2773

Schlafly, E. F., \& Finkbeiner, D. P. 2011, ApJ, 737, 103

Schlegel, D. J., Finkbeiner, D. P., \& Davis, M. 1998, ApJ, 500, 525

Schulze, S., Malesani, D., Cucchiara, A., et al. 2014, A\&A, 566, A102

Schulze, S., Krühler, T., Leloudas, G., et al. 2016, ArXiv e-prints [arXiv: 1612.05978]

Smartt, S. J., Valenti, S., Fraser, M., et al. 2015, A\&A, 579, A40

Smith, M., Sullivan, M., D’Andrea, C. B., et al. 2016, ApJ, 818, L8
Sparre, M., \& Starling, R. L. C. 2012, MNRAS, 427, 2965

Stanek, K. Z., Matheson, T., Garnavich, P. M., et al. 2003, ApJ, 591, L17

Stanway, E. R., Levan, A. J., Tanvir, N., et al. 2015, MNRAS, 446, 3911

Starling, R. L. C., Wiersema, K., Levan, A. J., et al. 2011, MNRAS, 411, 2792

Starling, R. L. C., Page, K. L., Pe'Er, A., Beardmore, A. P., \& Osborne, J. P. 2012, MNRAS, 427, 2950

Strolger, L.-G., Riess, A. G., Dahlen, T., et al. 2004, ApJ, 613, 200

Sukhbold, T., Ertl, T., Woosley, S. E., Brown, J. M., \& Janka, H.-T. 2016, ApJ, 821,38

Sutherland, P. G., \& Wheeler, J. C. 1984, ApJ, 280, 282

Taddia, F., Fremling, C., Sollerman, J., et al. 2016, A\&A, 592, A89

Tanvir, N. R., Krühler, T., Wiersema, K., et al. 2016, GRB Coordinates Network, 20321, 1

Taubenberger, S., Pastorello, A., Mazzali, P. A., et al. 2006, MNRAS, 371, 1459

Thompson, C., \& Duncan, R. C. 1993, ApJ, 408, 194

Thöne, C. C., Fynbo, J. P. U., Östlin, G., et al. 2008, ApJ, 676, 1151

Thöne, C. C., de Ugarte Postigo, A., Fryer, C. L., et al. 2011, Nature, 480, 72

Thöne, C. C., Christensen, L., Prochaska, J. X., et al. 2014, MNRAS, 441, 2034

Toy, V. L., Cenko, S. B., Silverman, J. M., et al. 2016, ApJ, 818, 79

de Ugarte Postigo, A., Blazek, M., Janout, P., et al. 2014a, Proc. of SPIE, 9152, 91520B

de Ugarte Postigo, A., Thöne, C. C., Rowlinson, A., et al. 2014b, A\&A, 563, A62

de Ugarte Postigo, A., Cano, Z., Perley, D. A., et al. 2015, GRB Coordinates Network, 18213, 1

de Ugarte Postigo, A., Cano, Z., Izzo, L., et al. 2016, GRB Coordinates Network, 20342, 1

Usov, V. V. 1992, Nature, 357, 472

Valenti, S., Benetti, S., Cappellaro, E., et al. 2008, MNRAS, 383, 1485

Vergani, S. D., Salvaterra, R., Japelj, J., et al. 2015, A\&A, 581, A102

Vernet, J., Dekker, H., D’Odorico, S., et al. 2011, A\&A, 536, A105

Volnova, A. A., Pruzhinskaya, M. V., Pozanenko, A. S., et al. 2017, MNRAS, 467, 3500

Volnova, A., Mazaeva, E., Inasaridze, R., et al. 2017, GRB Coordinates Network, 20442, 1

Wang, L., \& Wheeler, J. C. 2008, ARA\&A, 46, 433

Wang, L.-J., Cano, Z., Wang, S.-Q., et al. 2017, ArXiv e-prints [arXiv: 1702.03156]

Wheeler, J. C., Johnson, V., \& Clocchiatti, A. 2015, MNRAS, 450, 1295

Willingale, R., Starling, R. L. C., Beardmore, A. P., Tanvir, N. R., \& O'Brien, P. T. 2013, MNRAS, 431,394

Wilms, J., Allen, A., \& McCray, R. 2000, ApJ, 542, 914

Woosley, S. E. 1993, ApJ, 405, 273

Woosley, S. E., \& Bloom, J. S. 2006, ARA\&A, 44, 507

Xiao, H., Hajdas, W., \& Marcinkowski, R. 2016, GRB Coordinates Network, 20331, 1

Zafar, T., Watson, D., Fynbo, J. P. U., et al. 2011, A\&A, 532, A143

Zeh, A., Klose, S., \& Hartmann, D. H. 2004, ApJ, 609, 952

Zhang, B., \& Mészáros, P. 2001, ApJ, 552, L35 


\section{Appendix A: The radioactive-heating model}

The radioactive-heating model used in this work is based on the original analytical model of Arnett (1982). Since this seminal work, the basic model has been extended to include not only energy deposited via the radioactive decay of nickel, but also radioactive cobalt (Valenti et al. 2008). A further amendment to the model was made by Chatzopoulos et al. (2011) to include a term that considers the leakage of $\gamma$-rays into space, and hence not depositing this energy into the expanding SN ejecta.

In the original Arnett (1982) model there were several assumptions, many of which are still contained in the analytical model used here, which include:

1. a homologous expansion $\left(t^{-2}\right.$ scaling) of the ejecta;

2. spherical symmetry;

3. a photosphere that has a unique position in space;

4. the radioactive material present in the ejecta is located at the centre of the explosion and does not mix;

5. radiation-pressure dominance;

6. a small initial radius before explosion $\left(R_{0} \rightarrow 0\right)$;

7. the applicability of the diffusion approximation for photons (i.e. the photospheric phase).

Caveats of these assumptions, and their effect on the resultant modelling results can be found in Cano (2013).

The luminosity of a type I SNe as a function of time is:

$L(t)=M_{\mathrm{Ni}} \mathrm{e}^{-x^{2}}\left(\left(\epsilon_{\mathrm{Ni}}-\epsilon_{\mathrm{Co}}\right) \int_{0}^{x} A(z) \mathrm{d} z+\epsilon_{\mathrm{Co}} \int_{0}^{x} B(z) \mathrm{d} z\right)\left(1-\mathrm{e}^{-C t^{-2}}\right)$

where

$A(z)=2 z \mathrm{e}^{-2 z y+z^{2}}, B(z)=2 z \mathrm{e}^{-2 z y+2 z s+z^{2}}$

and $x \equiv t / \tau_{m}, y \equiv \tau_{m} /\left(2 \tau_{N i}\right)$, and $s \equiv\left(\tau_{m}\left(\tau_{\mathrm{Co}}-\tau_{\mathrm{Ni}}\right) /\left(2 \tau_{\mathrm{Co}} \tau_{\mathrm{Ni}}\right)\right)$.

The factor $\left(1-\mathrm{e}^{-C t^{-2}}\right)$ takes into consideration the possibility that some of the $\gamma$-rays produced during the radioactive decays escape directly into space, and hence do not interact with the SN ejecta. Small values of $C$ imply that most of the $\gamma$-rays escape into space. The $\gamma$-ray optical depth of the ejecta is $\tau=\kappa_{\gamma} \rho R=$ $C t^{-2}$, and hence the $\gamma$-ray opacity is $\kappa_{\gamma}=\left(4 \pi C v_{\mathrm{ph}}^{2}\right) /\left(3 M_{\mathrm{ej}}\right)$.

The energy release in one second by one gram of ${ }^{56} \mathrm{Ni}$ and ${ }^{56} \mathrm{Co}$ are, respectively, $\epsilon_{\mathrm{Ni}}=3.90 \times 10^{10} \mathrm{erg} \mathrm{s}^{-1} \mathrm{~g}^{-1}$ and $\epsilon_{\mathrm{Co}}=6.78 \times 10^{9} \mathrm{erg} \mathrm{s}^{-1} \mathrm{~g}^{-1}$ (Sutherland \& Wheeler 1984; Cappellaro et al. 1997). The decay times of ${ }^{56} \mathrm{Ni}$ and ${ }^{56} \mathrm{Co}$, respectively, are $\tau_{\mathrm{Ni}}=8.77$ days (see Taubenberger et al. 2006 and references therein) and $\tau_{\mathrm{Co}}=111.3$ days (Martin 1987).

$\tau_{m}$ is the effective diffusion time and determines the overall width of the bolometric light curve. $\tau_{m}$ is expressed in relation to the opacity $\kappa$ and the ejecta mass $\mathrm{M}_{\mathrm{ej}}$, as well as the photospheric velocity $v_{\mathrm{ph}}$ at the time of bolometric maximum:

$\tau_{m} \approx\left(\frac{\kappa}{\beta c}\right)^{1 / 2}\left(\frac{M_{\mathrm{ej}}}{v_{\mathrm{ph}}}\right)^{1 / 2}$

where $\beta \approx 13.8$ is a constant of integration (Arnett 1982), and $c$ is the speed of light. Additionally, we assume a constant opacity $\kappa=0.07 \mathrm{~cm}^{2} \mathrm{~g}^{-1}$ (e.g. Chugai 2000), which is justified if electron scattering is the dominant opacity source (e.g. Chevalier 1992). Finally, the kinetic energy of the ejecta is simply $E_{\mathrm{k}}=\frac{1}{2} M_{\mathrm{ej}} v_{\mathrm{ph}}^{2}$.

\section{Appendix B: The magnetar model}

The magnetar model used here is identical to that employed in Cano et al. (2016), in which the complete derivation of the model can be consulted. For the sake of completeness, we represent the main features of the model here.

The model considers three phases: (1) an AG component arising from the initial collision of the GRB ejecta with the surrounding medium; (2) a magnetar-powered AG phase; and (3) a magnetar-powered SN phase.

Phase (1) is modelled as a SPL (e.g. Rowlinson et al. 2013; Cano et al. 2015), which is analogous to the impulsive energy input term in the model of Zhang \& Mészáros (2001):

$L_{\mathrm{SPL}}(t)=\Lambda t^{-\alpha}\left(\mathrm{erg} \mathrm{s}^{-1}\right)$

where $\Lambda$ is the normalisation constant and $\alpha$ is the decay constant. Here we assume $\alpha=\Gamma_{\gamma}+1$, where $\Gamma_{\gamma}$ is the photon index of the prompt emission, assuming that the decay slope is governed by the curvature effect (e.g. Kumar \& Panaitescu 2000; Piran 2004).

The magnetar-powered AG (which persists as long as the jet remains collimated enough to deposit energy into the expanding fireball at large radii, and not into the expanding $\mathrm{SN}$ ) is modelled as a form of continuous energy input (Zhang \& Mészáros 2001). The general idea here is a magnetar central engine that deposits Poynting flux dominated dipole radiation into the ejecta (e.g. Dall'Osso et al. 2011) as:

$L_{\mathrm{AG}}(t)=L_{0}\left(1+\frac{t}{T_{0}}\right)^{-2}\left(\mathrm{erg} \mathrm{s}^{-1}\right)$

where $L_{0}$ is the plateau luminosity, $T_{0}$ is the plateau duration. In order to reduce the amount of free-parameters we have assumed a canonical NS with a mass of $1.4 M_{\odot}$ and a radius of $10^{6} \mathrm{~cm}$, and assumed a braking index of $n=3$ (see Lasky et al. 2017).

Once the jet spreads, it can no longer maintain a hole in the expanding ejecta, and instead it deposits its energy more locally in the $\mathrm{SN}$ itself. The analytical prescription used here is based on the previous works of Ostriker \& Gunn (1971), Kasen \& Bildsten (2010), Barkov \& Komissarov (2011) and Chatzopoulos et al. (2011). A magnetar-powered SN is expressed as:

$L_{\mathrm{SN}}(t)=\frac{E_{\mathrm{p}}}{t_{\mathrm{p}}} \exp \left(\frac{-x^{2}}{2}\right) \int_{0}^{x} \frac{z \exp \left(\frac{z^{2}}{2}\right)}{(1+y z)^{2}} \mathrm{~d} z \quad\left(\operatorname{erg~s}^{-1}\right)$

where $E_{\mathrm{p}}$ is the initial energy of the magnetar (units of erg) and $t_{\mathrm{p}}$ is the characteristic spin-down time of the magnetar (units of days). Additionally, $x=t / t_{\text {diff }}$ and $y=t_{\text {diff }} / t_{\mathrm{p}}$, where $t_{\text {diff }}$ is the diffusion timescale of the SN in units of days. As in the magnetar-powered AG phase, the radius of the magnetar is assumed to be $10^{6} \mathrm{~cm}$ (i.e. $10 \mathrm{~km}$ ), and we considered an $l=2$ magnetic dipole.

From these models we can determine the initial spin-period $(P)$ and magnetic-field strength $(B)$ of the magnetar central engine:

$B=\sqrt{\frac{1.3 \times 10^{2} P^{2}}{t_{\mathrm{p}, \mathrm{yr}}}}\left(10^{15} \mathrm{G}\right)$

and

$P=\sqrt{\frac{2 \times 10^{46}}{E_{\mathrm{p}}}}(\mathrm{ms})$, 
Table C.1. GRB 161219B/SN 2016jca: Spectroscopy observation log.

\begin{tabular}{ccccc}
\hline \hline UT date & $t-t_{0}(\text { day })^{a}$ & Range $(\AA)$ & Equipment & Exposure time (s) \\
\hline 21-Dec-2016 & 1.504 & $3200-22000$ & VLT-XS & $4 \times 600$ \\
26-Dec-2016 & 7.245 & $3700-7800$ & GTC-OSIRIS & $3 \times 900$ (in R1000B) \\
01-Jan-2017 & 13.253 & $3700-9300$ & GTC-OSIRIS & $2 \times 600$ (in R1000B and R1000R each) \\
03-Jan-2017 & 15.455 & $3985-9315$ & NTT-EFOSC2 & $2 \times 2700$ (grism 13) \\
09-Jan-2017 & 21.253 & $3700-9300$ & GTC-OSIRIS & $2 \times 900$ (in R1000B and R1000R each) \\
16-Jan-2017 & 28.237 & $3700-9300$ & GTC-OSIRIS & $2 \times 900$ (in R1000B and R1000R each) \\
22-Jan-2017 & 34.201 & $3700-7800$ & GTC-OSIRIS & $4 \times 900$ (in R1000B) \\
26-Jan-2017 & 38.178 & $3700-9300$ & GTC-OSIRIS & $2 \times 1200$ (in R1000B and R1000R each) \\
08-Feb-2017 & 51.144 & $3700-9300$ & GTC-OSIRIS & $2 \times 1200$ (in R1000B and R1000R each) \\
28-Feb-2017 & 71.089 & $3700-7800$ & GTC-OSIRIS & $2 \times 1200$ (in R1000B) \\
\hline
\end{tabular}

Notes. ${ }^{(a)}$ UT start time.

where $t_{\mathrm{p}, \mathrm{yr}}$ is the characteristic spin-down time of the magnetar in units of years.

These three phases are combined into a single model:

$L_{\mathrm{total}}(t)=L_{\mathrm{AG}}+\Phi L_{\mathrm{SN}}+L_{\mathrm{SPL}}\left(\mathrm{erg} \mathrm{s}^{-1}\right)$

where $\Phi$ is an additional free-parameter that was fit to the optical LCs. Therefore, if a GRB-SN bump has a value of $\Phi \approx 1$, this event can be considered as being powered entirely by EM emission from a magnetar central engine. Conversely, for all events where $\Phi>1$, additional sources of heating are needed to explain the luminosity of the SN phase, which is likely due to the heating from the radioactive decay of nickel and cobalt into their daughter products.

\section{Appendix C: Spectroscopic observation Log}

A summary of our spectroscopic observations are given in Table C.1.

\section{Appendix D: Photometry}

Our optical/NIR photometry is presented in Table D.1. All magnitudes are of the AG+SN+host galaxy, are uncorrected for extinction, and are for a $2^{\prime \prime} .2$ circular aperture centred on the position of the OT. Magnitudes in filters griz are in the AB system, while those in filters $J H K$ are in Vega. 
Z. Cano et al.: GRB 161219B/SN 2016jca: A low-redshift gamma-ray burst supernova powered by radioactive heating

\&

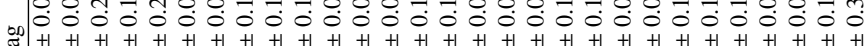

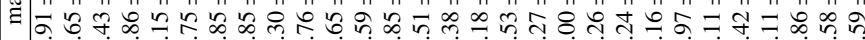

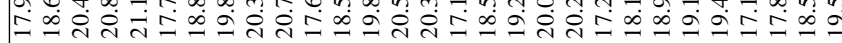

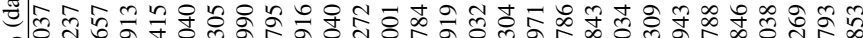

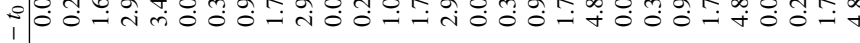

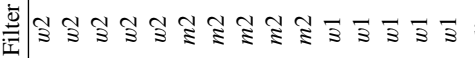

\begin{tabular}{l|l}
0 \\
0
\end{tabular}

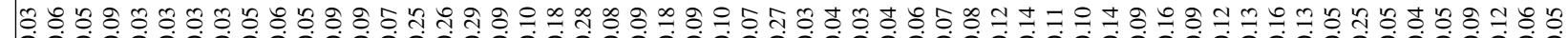

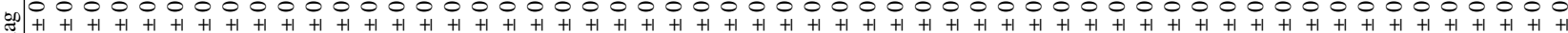

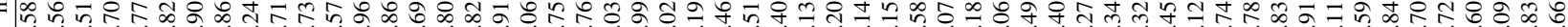

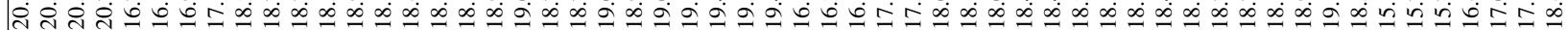

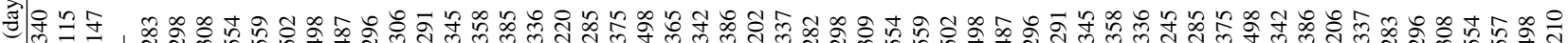

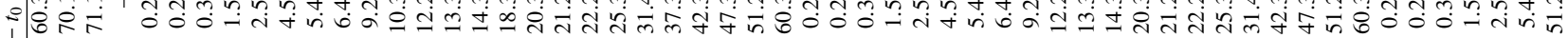

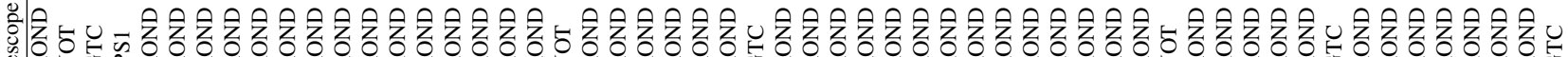
焉

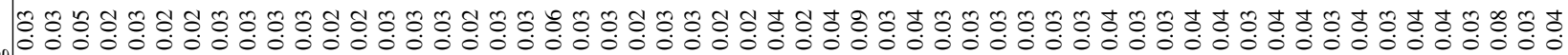

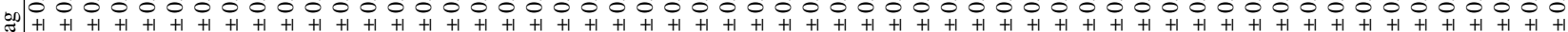

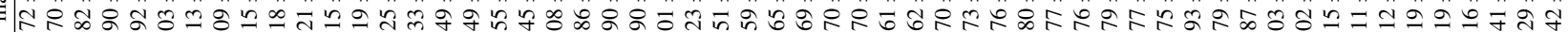

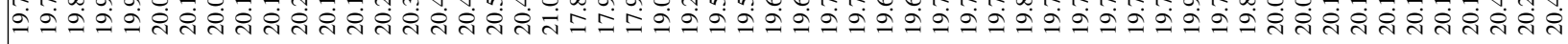

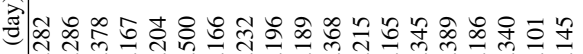

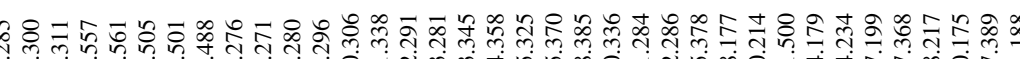
1 $\overrightarrow{\mathrm{s}}$

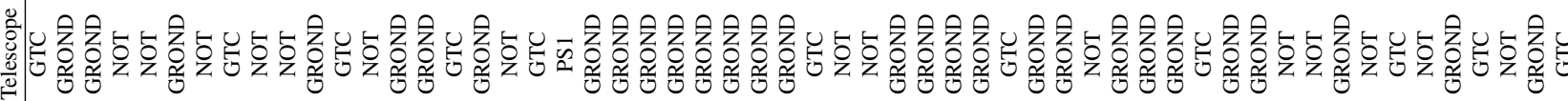
ธุ ธุ

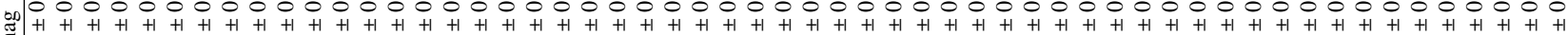
\#

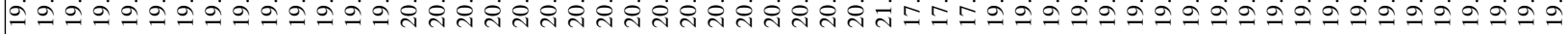

通施 $\because 0$ ป

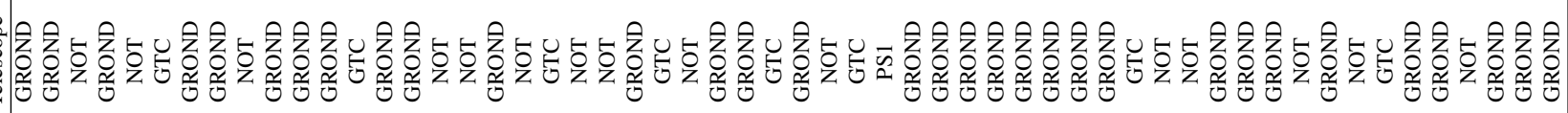

궁

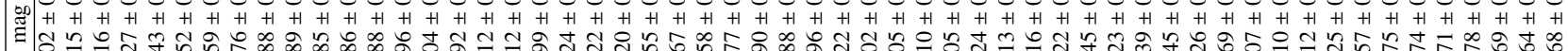
送

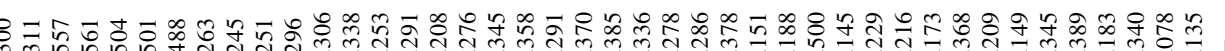

